\title{
Behavioral Evidence of Trigeminal Neuropathic Pain Following Chronic Constriction Injury to the Rat's Infraorbital Nerve
}

\author{
Bart P. Vos, ${ }^{a}$ Andrew M. Strassman, and Raymond J. Maciewicz \\ Pain Physiology Laboratory, Department of Neurology, Massachusetts General Hospital and Harvard Medical School, \\ Charlestown, Massachusetts 02129
}

Video recordings of free behavior and responses to mechanical facial stimulation were analyzed to assess whether chronic constriction injury $(\mathrm{CCl})$ to the rat's infraorbital nerve (loN) results in behavioral alterations indicative of neuropathic pain. A unilateral $\mathrm{CCl}$ was produced by placing loose chromic gut ligatures around the loN.

After $\mathbf{C C l}$ to the loN, rats exhibited changes in both nonevoked and evoked behavior. Behavioral changes developed in two phases. Early after $\mathrm{CCl}$ (postoperative days 115), rats showed increased face-grooming activity with facewash strokes directed to the injured nerve territory, while the responsiveness to stimulation of this area was decreased. Later after $\mathrm{CCl}$ (postoperative days 15-130), the prevalence of asymmetric face grooming was reduced but remained significantly increased compared to control rats. The early hyporesponsiveness was abruptly replaced by an extreme hyperresponsiveness: all stimulus intensities applied to the injured nerve territory evoked the "maximal" response (brisk head withdrawal, avoidance behavior plus directed face grooming). This response was never observed in control rats. Concurrently, IoN ligation rats showed a limited increase in the responsiveness to stimulation of the contralateral loN territory, and around postoperative days 30-40 the responsiveness to stimulation of facial areas outside the ION territories also increased. The hyperresponsiveness to stimulation of the ligated IoN territory slightly decreased from $60 \mathrm{~d}$ postoperative.

Throughout the study, loN ligation rats showed decreased exploratory behavior, displayed more freezing-like behavior, had a slower body weight gain, and a higher defecation rate, compared to control rats.

The behavioral alterations observed after $\mathrm{CCl}$ to the loN are indicative of severe sensory disturbances within the territory of the injured nerve: mechanical allodynia develops after a period of relative hypo-/anesthesia during which behavioral signs of recurrent spontaneous, aversive (possibly painful) sensations (paresthesias/dysesthesias) are maximal.

\footnotetext{
Received Feb. 10, 1993; revised Sept. 17, 1993; accepted Oct. 19, 1993.

This work was supported in part by Grant NS24594 from the National Institute of Neurological Disorders and Stroke (NIH).

Correspondence should be addressed to Andrew Strassman, Ph.D., Department of Neurobiology, Harvard Medical School, 220 Longwood Avenue, Boston, MA 02115 .

Present address: Laboratory of Anesthesiology, Universitaire Instelling Antwerpen, Universiteitsplein 1, B-2610 Wilrijk-Antwerpen, Belgium.

Copyright (C) 1994 Society for Neuroscience $0270-6474 / 94 / 142708-16 \$ 05.00 / 0$
}

[Key works: peripheral nerve injury, trigeminal system, orofacial pain, mechanical allodynia, face-grooming behavior, hyperresponsiveness]

Damage to peripheral somatosensory nerves sometimes leads to a condition of persistent pain. Lesions of primary afferent fibers associated with persistent pain disorders can result from a variety of traumatic injuries or diseases (White and Sweet, 1969). Psychophysically, neuropathic pain syndromes are typified by (1) a severely disturbed stimulus-response function and (2) the presence of nonevoked, continuous, or recurring pain sensations in the cutaneous region innervated by the damaged nerve. The specific somatosensory disorders are commonly referred to as allodynia (innocuous somatosensory stimulation evokes abnormally intense, prolonged pain sensations with an explosive, radiating character) (Kugelberg and Lindblom, 1959; Lindblom and Verillo, 1979), hyperalgesia (noxious stimulation evokes more intense and prolonged pain sensations) (Merskey, 1986; Campbell et al., 1988; Lindblom, 1990), paresthesia (spontaneous aversive but nonpainful sensations), and dysesthesia (spontancous pain sensations) (Mitchell, 1872; Noordenbos, 1959).

Research efforts to elucidate the etiology and pathophysiology of neuropathic pain disorders have been centered around a series of behavioral animal models (Lombard et al., 1979; Wall et al., 1979; Coderre et al., 1986; Bennett and Xie, 1988; Attal et al., 1990; Seltzer et al., 1990; Kim and Chung, 1992). In most of these models, experimental lesions of primary afferent fibers were induced by a variety of procedures including transection of a tightly ligated nerve (neuroma model) (Wall et al., 1979), dorsal rhizotomy (deafferentation models) (Lombard et al., 1979), and chronic constriction of a nerve trunk or part of it $(\mathrm{CCI})$ (Bennett and Xie, 1988; Attal et al., 1990; Seltzer et al., 1990; Kim and Chung, 1992).

So far, behavioral changes observed following CCI to the sciatic nerve have been most reminiscent of the clinical condition of neuropathic pain. Rats with a CCI to the sciatic nerve showed behavioral signs of (1) allodynia (reduced vocalization thresholds for mechanical stimulation of the foot, reluctance to place the foot down on a normal or innocuously cold surface), (2) hyperalgesia (reduced withdrawal latency, prolonged flexion of the hind paw and the occasional occurrence of hind limb flails or intense licking of the affected paw in response to noxious thermal, mechanical, or chemical stimulation of the plantar surface of the foot), and (3) paresthesias or dysesthesias (disturbed gait, episodes of hind paw licking, and "hind paw scratching," occasional autotomy) (Bennett and Xie, 1988; Attal et al., 
1990; Seltzer et al., 1990; Kim and Chung, 1992; Kupers et al., 1992; Ro and Jacobs, 1993). In the majority of these studies, observed behavioral abnormalities started $2-5 \mathrm{~d}$ following the ligation and peaked circa postoperative day 14 . However, the interpretation of the observed behavioral changes following CCI to the sciatic nerve is complicated by the fact that (1) the common sciatic nerve also includes a significant motor component (Bennett and Hargreaves, 1990; Hirata et al., 1990; Schouenborg and Kalliomäki, 1990; Ro and Jacobs, 1993; also De Medinaceli et al., 1982; Kerns et al., 1991) and (2) the saphenous nerve is believed to be involved in the behavioral effects of sciatic nerve damage (Devor et al., 1979; Ro and Jacobs, 1993).

The aim of this study is to investigate whether $\mathrm{CCI}$ to a pure sensory nerve in the rat, that is, the infraorbital nerve (IoN), results in behavioral changes that indicate the presence of painful sensory disturbances in the facial region innervated by the injured nerve. The IoN forms almost the entire maxillary division of the trigeminal nerve in the rat (Greene, 1955). Its territory includes the mystacial vibrissae, the hairy skin surrounding them (vibrissal pad), part of the rhinarium, the upper teeth, and part of the dorsal part of the oral cavity (Vincent, 1913; Greene, 1955; Fink et al., 1975). Morphologically, the IoN consists of $18-25$ fascicles containing $\pm 20,000$ myelinated $(60 \%)$ and $\pm 13,000$ unmyelinated $(40 \%)$ fibers, evenly distributed through the nerve (Jacquin et al., 1984). Behavioral studies in which the IoN was transected or anesthetized have demonstrated its importance for facial tactile and pain sensation (Gregoire and Smith, 1975; Welle and Coover, 1978; Hofer et al., 1981; Miller, 1981; Jacquin and Zeigler, 1983, 1984; Kenyon et al., 1983; Zeigler et al., 1984; Berridge and Fentress, 1986; Klein et al., 1991; Stern and Kolunie, 1991).

Clinical studies have suggested that lesions to trigeminal afferent fibers more often lead to conditions of persistent pain than similar lesions of spinal nerves (Sweet, 1984). In addition, the clinical features of the most frequently observed persistent facial pain syndromes that are believed to be related to trigcminal nerve damage (i.e., trigeminal neuralgia and anesthesia dolorosa) differ from those observed in pain syndromes associated with spinal nerve damage (Kugelberg and Lindblom, 1959; Gregg et al., 1979; Fromm et al., 1984). Some authors have therefore hypothesized that the trigeminal system is more prone to develop neuropathic pain due to its peculiar anatomo-physiological organization (Kerr, 1970; Sweet, 1984).

The present article reports an extensive analysis of spontaneous behavior and of responses to mechanical stimulation of the face observed after CCI to the IoN. Previous reports on behavioral consequences of trigeminal nerve injury (Anderson et al., 1971; Burchiel, 1980), as well as studies of the behavioral responses to chemical irritation of the trigeminal nucleus caudalis (King et al., 1956; King and Barnett, 1957; King, 1970; Black, 1974; Kryzhanovsky et al., 1974; Sakai et al., 1979), both in rats and in cats, have described the occurrence of recurrent attacks of ipsilateral face grooming together with hyperresponsiveness to light mechanical stimulation of the ipsilateral side of the face. Experimental studies of acute trigeminal pain in rats commonly report ipsilateral face grooming as the most obvious behavioral response to noxious stimulation of the face (Rosenfeld et al., 1978; Morris et al., 1982; Ramabadran and Bansinath, 1986; Clavelou et al., 1989; Cahusac et al., 1990). In view of the association of altered face-grooming patterns with the presence of facial pain, changes in face-grooming behavior accurring in rats with an IoN ligation were analyzed in great detail. The mechanical stimulation test consisted of a noninvasive procedure in freely moving rats in which responses were quantified using a semiparametric scoring system.

Preliminary results of this study were previously reported (Vos and Maciewicz, 1991).

\section{Materials and Methods}

\section{Ethical concerns}

Animals were treated and cared for according to the ethical standards and guidclincs for investigations of experimental pain in animals prescribed by the Committee for Research and ethical issues of the International Association for the Study of Pain (1983). Considering the possibility of a discomforting disorder, the number of animals studied was restricted to the bare minimum necessary to uphold the power of the statistical analysis $(N=8$ per group for effect size $=1.5 \mathrm{SD} ; \alpha=0.5$; power $=0.80$ ) (Hinkle and Oliver, 1983). All animals were used in all tests. The prolonged study period presented here was only done after pilot studies of short duration and was deemed necessary to describe the time course of observed behavioral changes. Procedures for handling and testing described in this study were reviewed and approved by the Animal Care Committee at the Massachusetts General Hospital in accordance with federal laws.

\section{Subjects}

Sixteen male Sprague-Dawley rats (Charles River; $250-300$ gm at arrival) were used. They were housed in plastic cages $(24 \times 41 \times 18 \mathrm{~cm}$ two or three rats per cage) under a partially reversed $12: 12 \mathrm{hr}$ dark/light cycle (lights on at $1500 \mathrm{hr}$ ) in a colony room with an ambient temperature of $23 \pm 2^{\circ} \mathrm{C}$. Water and food were available ad libitum.

Rats were randomly assigned to one of two groups. In eight animals the IoN was ligated on one side (left, $n=4$; right, $n=4$ ). Rats in the control condition ( $n=8)$ received a unilateral sham procedure (left, $n$ $=4$; right, $n=4$ ). A coding system was used to blind the investigator to the nature of the surgical procedure performed on individual animals.

\section{Surgery}

Rats were anesthetized with pentobarbital $(60 \mathrm{mg} / \mathrm{kg}$, i.p.) and (reated with atropine $(0.4 \mathrm{mg} / \mathrm{kg}$, i.p.). The head of the rat was fixed in a Narishige head holder and mounted in a Kopf stereotaxic frame. All surgery was performed under direct visual control using a Zeiss operation microscope (10-25x).

The intraorbital part of the IoN was exposed using a surgical procedure similar to that described by Gregg (1973) and Jacquin and Zeigler (1983). A midline scalp incision was made, exposing skull and nasal bone. The edge of the orbit, formed by the maxillary, frontal, lacrimal, and zygomatic bones, was dissected free. To give access to the IoN, orbital contents were gently deflected with a cotton-tipped wooden rod. The IoN was dissected free at its most rostral extent in the orbital cavity, just caudal to the infraorbital foramen. Two chromic gut (5-0) ligatures were loosely tied around the IoN ( $2 \mathrm{~mm}$ apart). To obtain the desired degree of constriction a criterion formulated by Bennett and Xie (1988) was applied: the ligations reduced the diameter of the nerve by a just noticeable amount and retarded, but did not occlude, circulation through the superficial vasculature. The scalp incision was closed in layers using nylon sutures (7-0). In rats of the control group, the IoN was exposed on one side using the same procedure. The exposed IoN was not ligated.

\section{Behavioral testing}

Rats were tested $3 \mathrm{~d}$ before the surgery and 3, 6, 9, 12, 15, 20, 25, 30 $40,50,60,80$, and $130 \mathrm{~d}$ after the surgery. Testing was conducted from 0700 to $1400 \mathrm{hr}$. Each rat was individually transported from the colony to the test room (5-8 min trip), in a covered cage without bedding. In the test room, white background noise $(50 \mathrm{~dB})$ was used to decrease interference of sudden auditory stimulation. Light was provided by two $60 \mathrm{~W}$ incandescent red bulbs suspended $1 \mathrm{~m}$ above the center of the test area, lighting the center but leaving the circumference relatively $\mathrm{dim}$ and the remainder of the room dark. Each test session consisted of three subsequent testing procedures: (1) observation of free behavior, (2) observation of thigmotactic scanning activity, and (3) assessment of the animal's responses to mechanical stimulation of the face. After testing, body weight was measured. 


\section{Observation of free behavior}

Rats were placed in a transparent plastic cage $(24 \times 35 \times 18 \mathrm{~cm})$ with a mirrored back. A video camera was placed $1 \mathrm{~m}$ in front of the cage and positioned so that the image of the rat's body covered at least onefourth of the recorded view. Videotaped observations were analyzed on two levels, as follows

(1) Time analysis of behavioral activity. To assess changes in general behavioral activity, observed behavior was scored as (1) exploratory bchavior (walking, running, climbing, rearing, and sniffing), (2) freezinglike behavior (freezing and immobile posture), (3) resting/sleeping (head resting on flexed forepaws with eyes open/closed), (4) face grooming (movement patterns in which paws contact facial areas), and (5) body grooming (paws, tongue, or incisors are brought in contact with a body area other than the face or the forepaws). Since neither food nor water was available in the testing cage, the categories drinking and eating were never observed. The total time spent in each of the behavioral categories was determined for each 7 min observation period, using an IBMcompatible microcomputer program for recording of behavior durations (Tarapacki and Kristal, 1990). This analysis was done on line.

(2) Structural analysis of face-grooming behavior. To analyze specific changes in face-grooming behavior, videotaped face-grooming episodes, recorded preoperatively and at 6 of the 13 postoperative time points $(3,9,15,30,60$, and $130 \mathrm{~d}$ postoperatively), were played back frame by frame or in slow motion ( $30 \mathrm{frames} / \mathrm{sec}$ ). Face-grooming actions were scored according to a scoring system similar to the ones described by Fentress (1972) and Berridge (1990).

Face-grooming actions. Fifteen face-grooming actions were identified. These included nine different types of "face wash strokes" by the paw over the face, which were differentiated according to the side (ipsilateral, contralateral, or bilateral) and the facial region to which they were directed (see Fig. 3A) (Berridge and Fentress, 1986). The point of initial forelimb contact determined the facial region to which a face-wash stroke was directed. Since face-wash strokes proceed forward and downward from the point of initial forelimb contact (see Fig. $3 A$ ), they were scored as (1) small, if they were restricted to the hairy skin below the rostroventral boundary of the mystacial vibrissae or the rhinarium; (2) medium, if they were directed to the hairy skin below the eye extending to the rostroventral border of the vibrissal pad, including the mystacial pad (i.e., the territory of the IoN); or (3) large, if they were directed to the hairy skin above the eye (Berridge, 1990). Laterality was scored either as unilateral left or unilateral right, if the stroke was performed with only one paw, or as bilateral with both paws in simultaneous trajectories. Asymmetrical strokes of unequal amplitude were scored as unilateral strokes of the dominant paw. In addition to these nine classes of "face-wash strokes," six other types of face-grooming actions were identified (also from Berridge, 1990): head shakes (rhythmic lateral motions of the head), forelimb flails (rapid "shaking" movements of the forepaw and limb), paw licks (forepaws are brought in contact with the tongue, which is repeatedly protruded), ear grasps (unilateral paw strokes of large amplitude in which the ear or surrounding fur is grasped and pulled toward the mouth), tongue protrusions (tongue exits the oral cavity visibly), and chin rubs (forepaws stroke simultaneously the ventral aspect of the head, in a caudal-rostral direction). All face-grooming actions were counted as separate units except for continuous paw licking, for which every $5 \mathrm{sec}$ was considered a separate unit.

Face grooming episodes. For each observation session a tabular record of the sequence of observed face-grooming actions was generated by entering identified actions and the time that they occurred (as determined by a calibrated video recorder clock) into a spreadsheet (STATVIEW Il 4.0, Abacus Inc.). In the rat, face grooming typically occurs in discrete episodes (Berridge, 1990). A face-grooming episode may be defined as an uninterrupted sequence of face-grooming actions. The tabular records were used to identify individual face-grooming episodes. In order to have an objective criterion for determining the end of a face-grooming episode, it was necessary to define the maximum interval between two sequential face-grooming actions that can be considered merely a pause (intraepisode space), rather than a complete interruption of face-grooming behavior (interepisode gap). The criterion to differentiate between intraepisode spaces and interepisode gaps was determined by generating a log survivor function of the durations of all intervals between facegrooming actions observed preoperatively (Fagen and Young, 1978). The criterion was $4 \mathrm{sec}$.

For each observation session, the total number of observed facegrooming episodes was counted. In addition, the following structural features of each individual face-grooming episode were determined: (1) duration, time in seconds, elapsed between the beginning of the first and the end of the last action of a sequence of face-grooming actions; (2) context and sequential variability: if a sequence of face-grooming actions was neither preceded nor followed by body grooming, the episode was categorized as spontaneous face grooming; if body grooming was present before or after a sequence of face-grooming actions, the episode was categorized as face grooming during body grooming. Facegrooming episodes occurring during body grooming were further subdivided into sequentially variable (BG-VAR) or sequentially stereotyped (BG-STE) face-grooming episodes, based on the sequential order of individual face-grooming actions (Berridge and Fentress, 1986; Berridge, 1990). Both sequentially variable and sequentially stereotyped face-grooming episodes consist of the same face-grooming actions but can be differentiated based on the order in which individual actions appear. Berridge (1990) has shown that sequentially stereotyped facegrooming episodes follow a rigid pattern of order of individual facegrooming actions, starting with a set of small bilateral face-wash strokes, performed rapidly $(6-7 \mathrm{~Hz})$ and appearing as elliptical strokes contacting the upper lip and the most ventrorostral border of the vibrissal pad, followed by a short series of slower bilateral strokes of ascending amplitude, with the final strokes passing above the ears, followed by a tucking of the head and twisting of the body, and terminated with a body-groom action during which the ventrolateral torso is licked. Facegrooming episodes occurring during body grooming in which this stereotyped sequential order does not occur were termed "sequentially variable." The sequential structure and the form of individual actions of scquentially stereotyped face-grooming episodes were found to be highly resistant to experimental procedures that disrupted cutaneous feedback from the face (trigeminal deafferentation, Berridge and Fentress, 1986).

\section{Thigmotactic scanning}

Immediately after the observation of free behavior, rats were placed near the center of an open field $(60 \times 60 \mathrm{~cm})$ surrounded by $40-\mathrm{cm}-$ high walls. Floor and walls were painted black. The box was scarcely illuminated by one $60 \mathrm{~W}$ incandescent red bulb suspended $2 \mathrm{~m}$ above the center of the floor. The rat's behavior was recorded for a 1 min period by a video camera positioned $1 \mathrm{~m}$ above the center of the field. The total time the rat moved along the wall of the field with the snout within a range of $5 \mathrm{~cm}$ to the wall (=thigmotaxis; Milani et al., 1989; Huston et al., 1990) was determined for both sides of the face using the same computer program for recording of behavior durations as mentioned above.

\section{Mechanical stimulation}

Stimuli. For mechanical stimulation a graded series of five von Frey hairs (Pressure Aesthesiometer, Stoelting Co, Chicago, IL) and a pin prick were used. Von Frey hairs consisted of plastic monofilaments of equal length $(4 \mathrm{~cm})$ and varying diameters for which the force required to bend each filament was, respectively, $\sim 1 \mathrm{gm}, \sim 2 \mathrm{gm}, \sim 4 \mathrm{gm}, \sim 9$ $\mathrm{gm}$, and $\sim 16 \mathrm{gm}$ (or converted to $\log$ units, respectively, $4.08,4.31$, $4.56,4.93$, and 5.22 units). A 24 gauge injection needle, bent in a $30^{\circ}$ angle and mounted on a syringe, was used to apply the pin prick stimulations. Pilot observations showed that all intensities evoked a behavioral response when applied to the face of normal animals. Intensities used in this study could therefore be considered above perceptual threshold. Each stimulation consisted of two consecutive placements (interval $<1 \mathrm{sec}$ ) of the stimulus hair or the pin prick.

Stimulated areas. Stimuli were applied (1) within the IoN territory, near the center of the vibrissal pad on hairy skin surrounding the mystacial vibrissae, or (2) outside the IoN territory, in the territory of the auriculotemporal nerve, on hairy skin $\sim 50 \mathrm{~mm}$ under the ear. These areas were stimulated on both sides of the face, ipsilateral and contralateral to the side where surgery was performed (see Fig. 1).

Testing procedures. For observation of responses to mechanical stimulation, rats were placed in a small transparent plastic cage $(14 \times 22$ $\times 16 \mathrm{~m}$ ). The test session was recorded on videotape with a video recorder placed approximately $50 \mathrm{~cm}$ in front of the cage. The observation cage was positioned at eye level of the experimenter, who sat quietly on the opposite side. Stimulations were delivered by reaching into the cage. To decrease the conspicuousness of the experimenter, light was provided by a $60 \mathrm{~W}$ indescendant red bulb suspended $1 \mathrm{~m}$ above the center of the test area, lighting the center but leaving the circumference relatively dim and the remainder of the room dark. Reaching movements were performed slowly. 
Before the actual stimulation session rats were adapted to the observation cage for $10 \mathrm{~min}$. During this period, the experimenter reached into the cage every $30 \mathrm{sec}$ to touch the walls of the cage with a plastic rod, similar to the ones on which the von Frey hairs are mounted. After the rats were habituated to the reaching movements, the series of mechanical stimulations was started. Stimulations were administered when the rat was in a sniffing/no locomotion state: with four paws placed on the ground, neither moving nor freezing, but exhibiting sniffing behavior. A new stimulus was applied only when the rat resumed this position and at least $30 \mathrm{sec}$ after the preceding stimulation.

During one session the complete series of von Frey hair intensities was presented in an ascending/descending series: $2 \mathrm{gm}, 9 \mathrm{gm}, 16 \mathrm{gm}, 4$ $\mathrm{gm}$, and $1 \mathrm{gm}$. The pin prick was always applied after completion of the von Frey hair series. All four facial areas were explored with one level of stimulation before using the next intensity. To counterbalance for order effects, facial areas were stimulated in a randomized order for each stimulus intensity, within each subject.

Response scoring. Responses were examined by playing videotaped reactions in slow motion (15 frames/sec) or in real time. Pilot observations indicated that a rat's response to mechanical facial stimulation consists of one or more of the following elements: (1) detection, rat turns head toward stimulating object, and stimulus object is then explored (sniffing, licking); (2) withdrawal reaction, rat turns head slowly away or pulls it briskly backward when stimulation is applied; sometimes a single face wipe, ipsilateral to the stimulated area occurs (note that a withdrawal reaction is assumed to include a "detection" element preceding the head withdrawal and therefore consists of two response elements) (see Table 1); (3) escape/attack, rat avoids further contact with stimulus object, either passively, by moving its body away from the stimulating object to assume a crouching position against cage wall, sometimes with the head buried under the body, or actively by attacking the stimulus object, making biting and grabbing movements; sometimes these actions are accompanied by vocalizations; (4) asymmetric face grooming, rat displays an uninterrupted series of at least three facewash strokes directed to the stimulated facial area.

The following rank-ordered descriptive response categories were formulated based on pilot observations: no response, mild aversive response, strong aversive response, prolonged aversive behavior (Table 1). Each category was given a score (0-4) based on the number of observed response elements. During pilot tests it appeared that with increasing stimulus intensities, more response elements were observed. The score was therefore assumed to reflect the magnitude of the aversiveness evoked by the mechanical stimulation.

Calculation of difference scores. Difference scores were introduced as an overall measure of altered responsiveness to mechanical facial stimulation. The difference score of an area on a particular postoperative day was calculated as the mean of the six differences (one for each stimulus intensity) between pre- and postoperative response scores evoked by stimulation of that area.

\section{Statistical analysis}

To assess potential baseline differences between the two groups, singlefactor analyses of variance (ANOVAs) were performed on preoperative data. Postoperative differences between groups and changes in postoperative measurements over time were analyzed with a mixed ANOVA with repeated measurements (RM-ANOVA). The Newman-Keuls method was used for post hoc multiple comparisons between groups. When visual inspection of the graphed data indicated potentially significant changes following sham operation, a paired two-tailed $t$ test was performed to confirm. Weight measures were also compared with a single-factor ANOVA. Spearman rank correlation coefficients and pointbiserial coefficients of correlation were calculated as descriptive measures of association. Due to technical failure, data of five rats (three IoN, two sham) gathered on postoperative day 25 were not recorded. This data point was therefore not considered in the statistical analyses. For graphical representations of means over time, the mean of day 25 was calculated based on data of the remaining rats.

Changes in response scores over the 14 test sessions (1 preoperativc and 13 postoperative) within each group were analyzed using Friedman one-way analysis of variance by ranks (Friedman ANOVA). Calculated $\chi^{2}$ values were corrected for ties (Friedman, 1937). When significant effects were found, multiple post hoc comparisons were performed to determine at which postoperative time points response scores were significantly different from preoperative scores. Changes in stimulus-response relations within each group were analyzed by calculating a Good-
Table 1. Response scoring system

\begin{tabular}{|c|c|c|c|c|c|}
\hline \multirow[b]{2}{*}{ Response category } & \multicolumn{4}{|c|}{$\begin{array}{l}\text { Observed response } \\
\text { elements }\end{array}$} & \multirow[b]{2}{*}{ Score } \\
\hline & $\begin{array}{l}\text { Detec- } \\
\text { tion }\end{array}$ & $\begin{array}{l}\text { With- } \\
\text { drawal }\end{array}$ & $\begin{array}{l}\text { Es- } \\
\text { cape/ } \\
\text { lattack }\end{array}$ & $\begin{array}{l}\text { Face } \\
\text { groom- } \\
\text { ing }\end{array}$ & \\
\hline No response & 0 & 0 & 0 & 0 & 0 \\
\hline Nonaversive response & 1 & 0 & 0 & 0 & 1 \\
\hline Mild aversive response & 1 & 1 & 0 & 0 & 2 \\
\hline Strong aversive response & 1 & 1 & 1 & 0 & 3 \\
\hline \multicolumn{6}{|l|}{ Prolonged aversive } \\
\hline behavior & 1 & 1 & 1 & 1 & 4 \\
\hline
\end{tabular}

man-Kruskal correlation coefficient between intensities and response scores. The stimulus-response relation was examined for three postoperative time periods: (1) 3-12 d postoperative (days $3,6,9,12$ ), (2) $15-40 \mathrm{~d}$ postoperative (days $15,20,30,40$ ), and (3) 50-130 d postoperative (days 50,60, 80, 130) (Goodman and Kruskal, 1979). It should be noted that the calculation of these correlation coefficients was based on a sample of only 24 of the 192 (eight rats $\times$ six intensities $\times$ four time points) responses observed during the respective periods, per group. This semi-independent sample consisted of a random selection of one response, of each animal on 3 of the $4 \mathrm{~d}$ of the time period under consideration. For determining the preoperative stimulus response relation, 24 of the 96 responses, observed in the total group, were randomly selected (not more than two responses per rat). Mann-Whitney $U$ tests were used for between-group comparisons of difference scores. For assessing statistical significance of resulting $U$ values, $Z$ values were corrected for ties.

\section{Results}

Nonevoked behavior

General behavioral activity

The most obvious bchavioral change that occurred following IoN ligation was a dramatic increase in face-grooming activity (Fig. 1A): IoN ligation rats exhibited significantly more face grooming than sham-operated rats [RM-ANOVA: $F(1,14)=$ $19.977, p<0.0005]$. Compared to preoperative or sham-operated rats, IoN ligation rats also showed a profound decrease of exploratory activity (Fig. $1 B$ ) [RM-ANOVA: $F(1,14)=19.888$, $p<0.0005]$ and spent more of the observation time in an immobile posture (Fig. 1C) [RM-ANOVA: $F(1,14)=7.277, p$ $<0.05]$. Only the amount of body grooming observed after IoN ligation remained unchanged compared to preoperative or shamoperated rats (Fig. $1 D$ ) [RM-ANOVA: $F(1,14)=3.435$, NS]. The increases in face-grooming and freezing-like behavior, and the concurrent decrease in exploratory behavior, observed in IoN ligation rats were maximal immediately after the operation and then waned over time, remaining significantly different from sham-operated rats throughout the $130 \mathrm{~d}$ study period [RMANOVA: time postoperative, respectively: $F(11,154)=2.912$, $p<0.05 ; F(11,154)=2.934, p<0.05 ; F(11,154)=4.032, p$ $<0.005$; group $\times$ time postoperative: all $F(11,154) \leq 1.562$, NS]. A sham operation alone did not significantly change postoperative behavioral activity (see Fig. $1 A-D$ for statistics). In IoN-ligation rats, only freezing-like behavior returned to a preopcrative level at approximately 2 months following ligation (Fig. 1C).

\section{Frequency and duration of observed face-grooming episodes}

To assess whether the increase in face-grooming activity was due to an increase of the number of face-grooming episodes or 


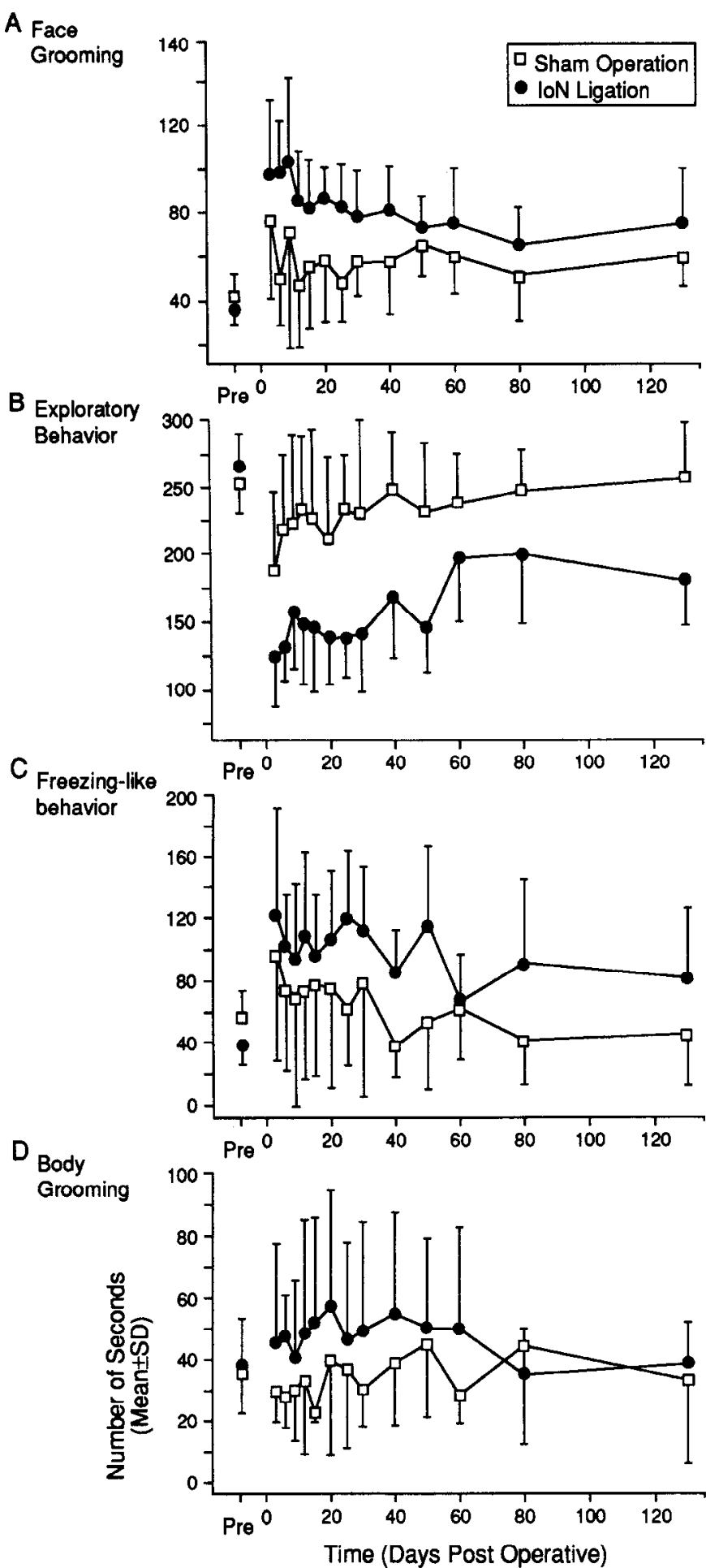

Figure 1. Time course of changes in behavioral activity. $A-D$, Line graphs representing the mean number of seconds that sham-operated rats (squares) or IoN ligation rats (circles) were observed executing face grooming $(A)$, exploratory behavior $(B)$, freezing-like behavior $(C)$, and body grooming $(D)$ during a 7 min observation session before the operation (Pre) and at 13 postoperative time points. Error bars indicate the standard deviation (SD). No significant baseline differences between groups were found before the operation for each of the behavioral categories [ANOVA, all $F(1,14) \leq 2.707$, NS]. Behavioral activity of shamoperated rats was never different from preoperative levels [ $t$ tests: -1.603 $<t(7)<1.342$, NS].
A

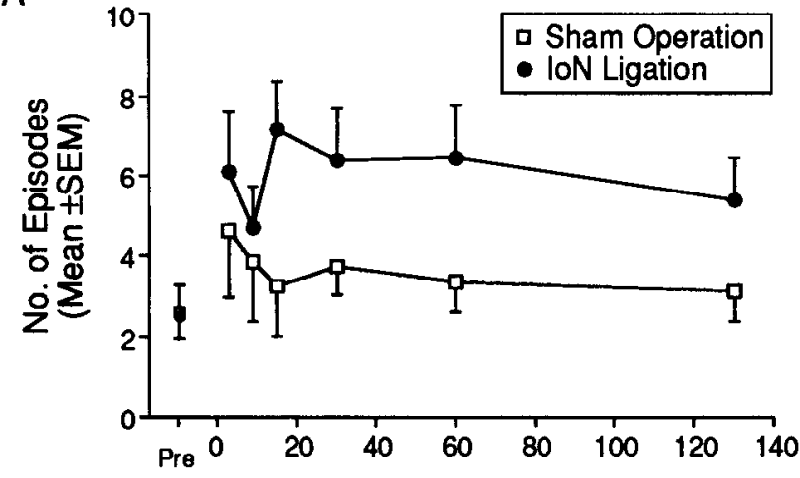

B

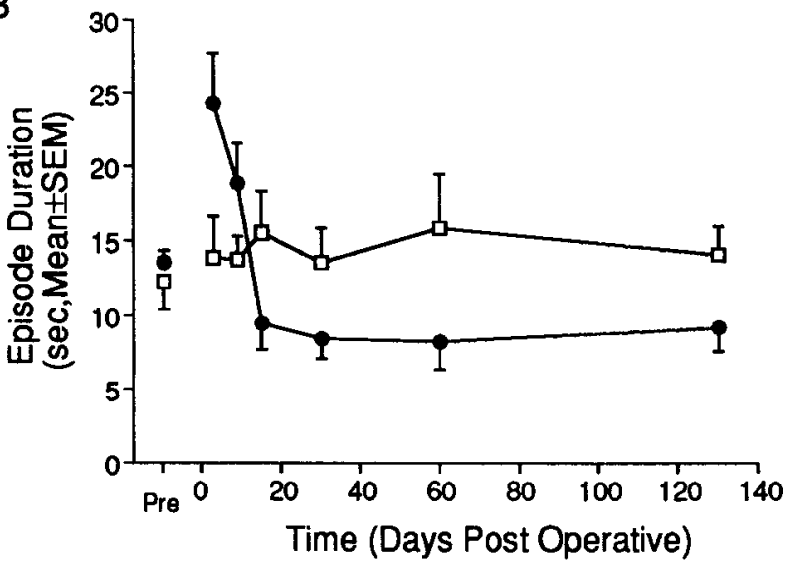

Figure 2. Frequency and duration of observed face-grooming episodes. $A$ and $B$, Line graphs representing the mean number of face-grooming episodes $(A)$ and their mean duration $(B)$ observed in sham-operated rats (squares) or IoN ligation rats (circles) before the operation (Pre) and at six postoperative time points. Error bars indicate the standard error of the means (SEM). Preoperatively, the incidence of face-grooming episodes and their mean duration were not different between the groups [ANOVA, both $F(1,14) \leq 0.188$, NS]. Frequency and duration of episodes in sham-operated rats were never different from preoperative levels [ $t$ tests; all $t(7)>1.059$, NS].

in the duration of the episodes, or both, the number of observed episodes and their mean duration were determined per rat for postoperative days $3,9,15,30,60$, and 130 . IoN ligation rats performed more face-grooming episodes than preoperative or sham-operated rats (Fig. 2A) [RM-ANOVA: $F(1,14)=13.394$, $p<0.005$ ]. This higher incidence of face-grooming episodes persisted throughout the $130 \mathrm{~d}$ postoperative period [RM-ANOVA: time postoperative, $F(5,70)=1.126$, NS; group $\times$ time postoperative, $F(5,70)=1.486$, NS] . Durations of face-grooming episodes observed in IoN ligation rats showed specific changes over time [RM-ANOVA: time postoperative, $F(5,70)=4.19$, $p<0.05$; group $\times$ time postoperative, $F(5,70)=5.346, p<$ 0.005 ]. Figure $2 B$ illustrates that during the first postoperative observation session, the duration increased compared to preoperative or sham-operated rats, while after postoperative day 15 , face-grooming episodes of IoN ligation rats decreased and remained significantly shorter than face-grooming episodes observed preoperatively or in sham-operated rats. In sham-operated rats, the postoperative episode frequency and duration was never different from the frequency and duration of facegrooming episodes observed preoperatively (see Fig. $2 B$ for statistics). 

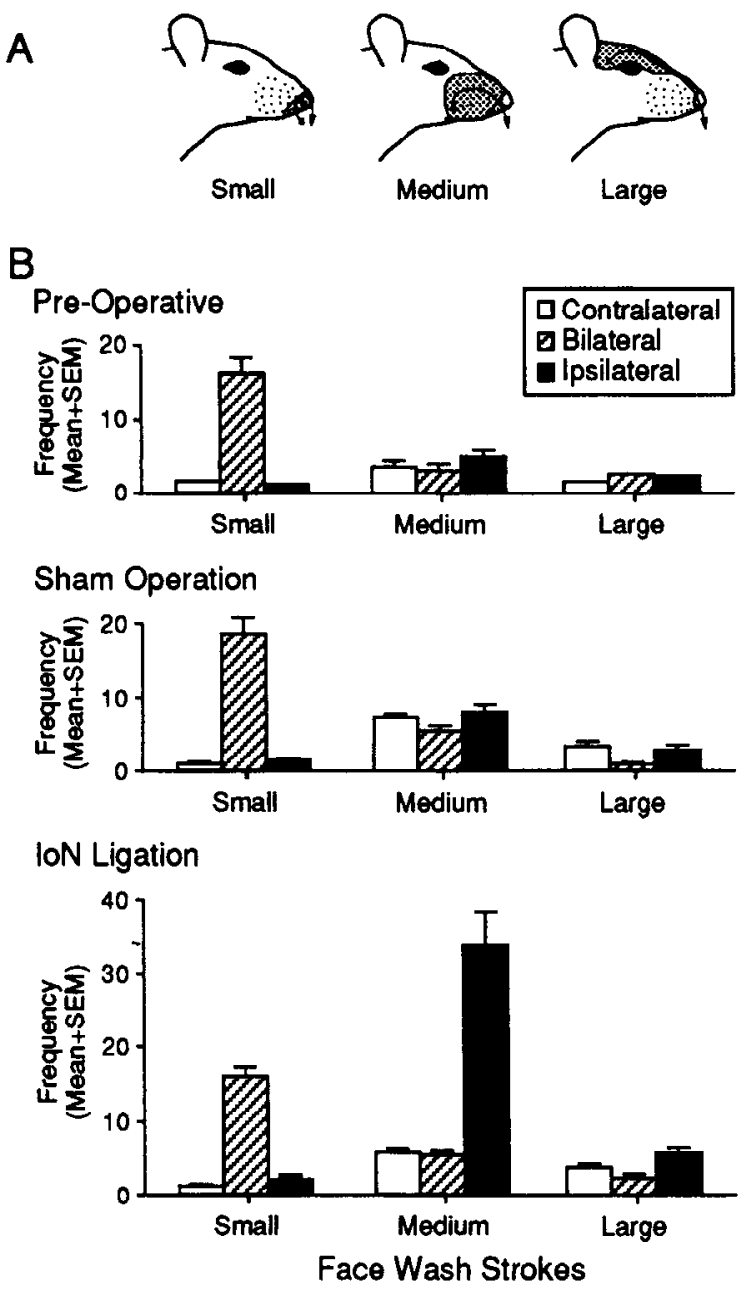

Figure 3. Distribution of face-wash strokes. $A$, Schematic drawing of small (left), medium (middle), and large (right) face-wash strokes (modified from Berridge and Fentress, 1986). The trajectories of the paw over the face are represented by arrows. The shaded areas indicate the facial region that is first contacted by the paw. This point of initial forelimb contact defines the amplitude of the face-wash stroke (see Materials and Methods). $B$, Histograms representing the frequency of small (left), medium (middle), and large (right) face-wash strokes, occurring contralateral to the surgery (open bars), bilateral (hatched bars), and ipsilateral to the surgery (solid bars) that were observed during a 7 min observation session in rats before the operation (top), in shamoperated rats (middle), and in IoN ligation rats (bottom). Mean values for sham-operated and IoN ligation rats were averaged over six postoperative time points $(3,9,15,30,60$, and $130 \mathrm{~d}$ postoperative). Error bars indicate the SEMs. Note the selective increase in the number of unilateral medium face-wash strokes ipsilateral to the surgery in IoN ligation rats.

\section{Changes in frequency and distribution of face-wash strokes}

Changes in facc-grooming bchavior werc also analyzcd on the level of individual face-wash strokes (Fig. $3 A$ ). The distribution of face-wash strokes over both sides of the face was analyzed to examine whether increased face-grooming activity observed in IoN ligation rats showed any gross structural abnormalities such as asymmetries or preferential grooming of specific facial regions.

Changes in the amount of face-grooming activity over time, described earlier (Fig. 1A), were also found for the total number of face-wash strokes [Spearman rho, $r(14)=0.92, p<0.05$ ]. IoN ligation rats displayed more face-wash strokes than pre-

\section{Sham Operation}

IoN Ligation

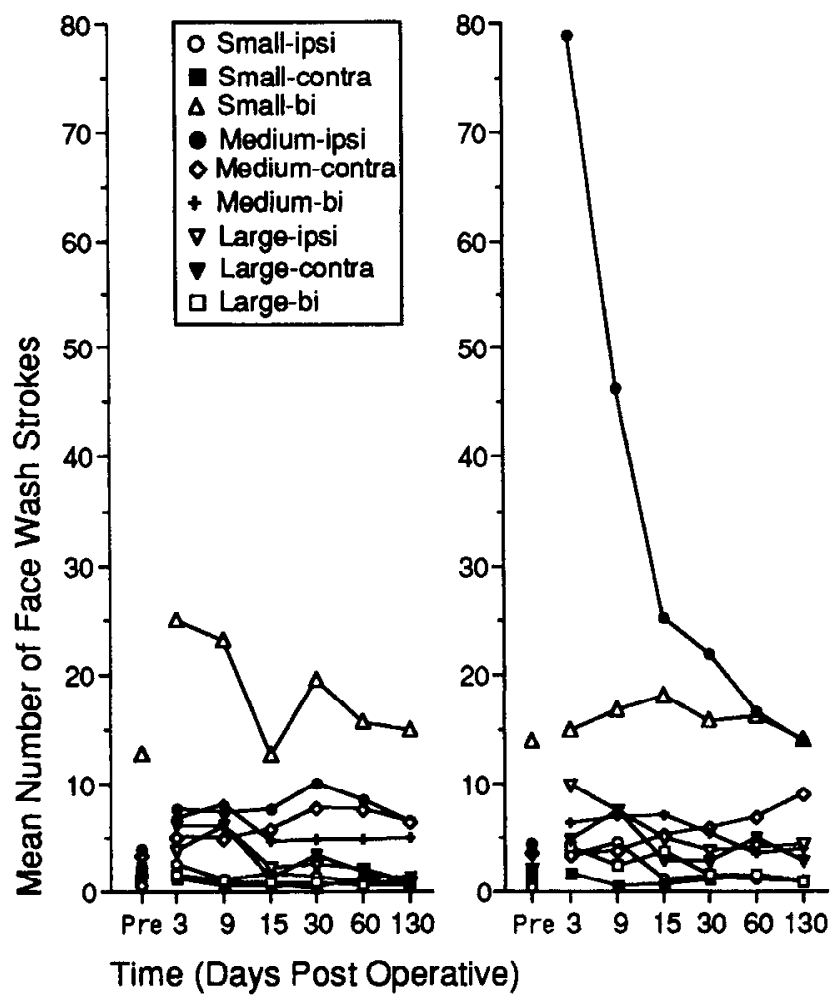

Figure 4. Frequency of face-wash strokes over time. Line graphs represent the mean number of each type of face-wash stroke (see legend for symbols) observed in sham-operated rats (left) and IoN ligation rats (right) before the operation (Pre) and at six postoperative time points. Preoperatively, the distribution of face-wash strokes (FWS) was not different between the groups [RM-ANOVA: group, $F(1,14)=0.040, p$ $=0.8442 ;$ FWS $\times$ group, $F(8,120)=0.9977, p=0.8131]$.

operative [ $t$ tests; all $t(7)<-5.405, p<0.0011]$ or shamoperated rats [RM-ANOVA: $F(1,14)=26.413, p<0.0005]$, and this increase in face-wash strokes waned over time [RMANOVA: time postoperative, $F(5,70)=7.3383, p<0.005$; group $\times$ time postoperative, $F(5,70)=3.814, p<0.05$ ]. The overall increase in the number of face-wash strokes observed in IoN ligation rats consisted almost entirely of an increase in medium-sized face-wash strokes, ipsilateral to the ligation (i.e., strokes directed to the territory of the ligated IoN) (Figs. 3B, 4) [RM-ANOVA: $F(1,14)=23.001, p<0.0005$ ]; there was no change in any of the other eight types of face-wash strokes, compared to preoperative or sham-operated rats (Figs. $3 B, 4$ ) [RM-ANOVA: all $F(1,14) \leq 3.056$, NS]. Figure 4 illustrates that the number of ipsilateral medium-sized face-wash strokes was maximal in the first $14 \mathrm{~d}$ following the operation and then decreased over time, but remained significantly greater than that in preopcrative or sham-opcrated rats throughout the $130 \mathrm{~d}$ observation period [RM-ANOVA: time postoperative, $F(5,70)$ $=4.069, p<0.05$; group $\times$ time postoperative, $F(5,70)=2.421$, $p<0.01]$.

\section{Comparisons between three types of face-grooming episodes}

Further analysis was carried out to determine whether the specific changes in face-grooming behavior observed in IoN ligation rats-more face-grooming episodes of an initially longer, subsequently shorter duration and asymmetrical distribution of the face-wash strokes, with more strokes directed to the territory of 
Sham Operation
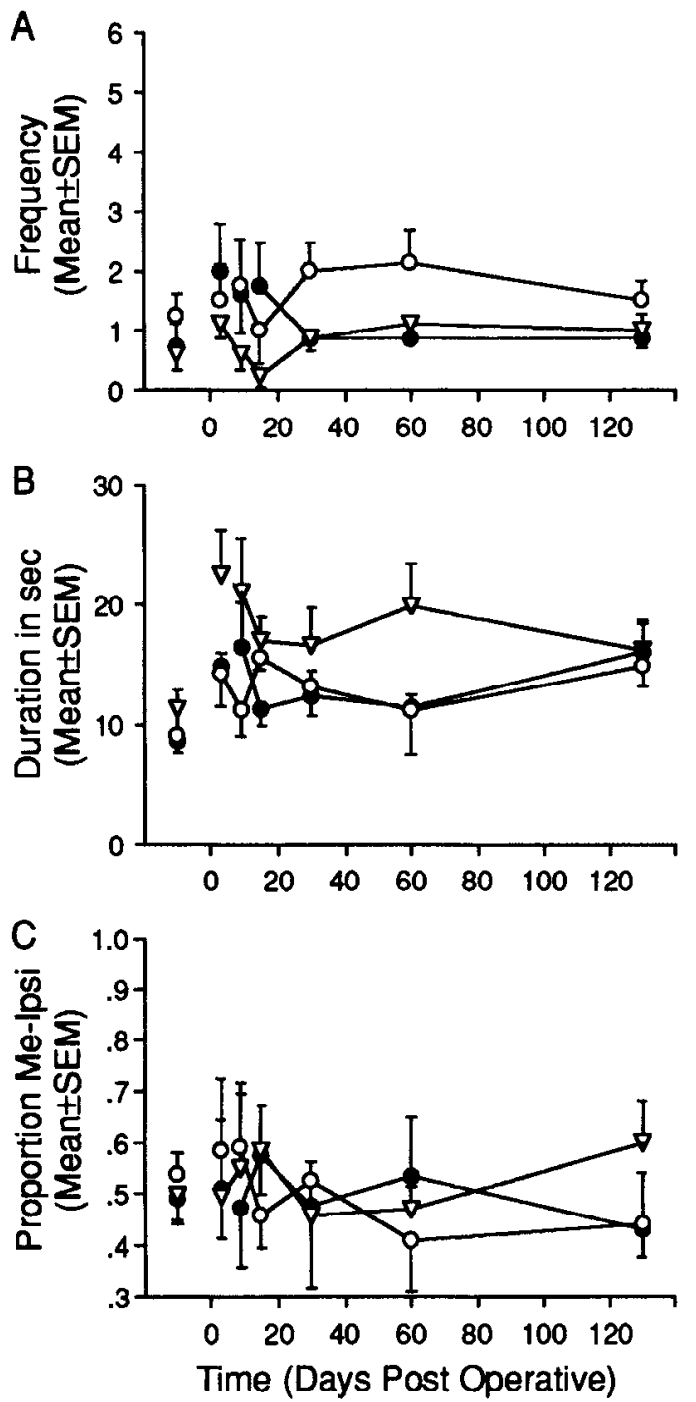

IoN Ligation
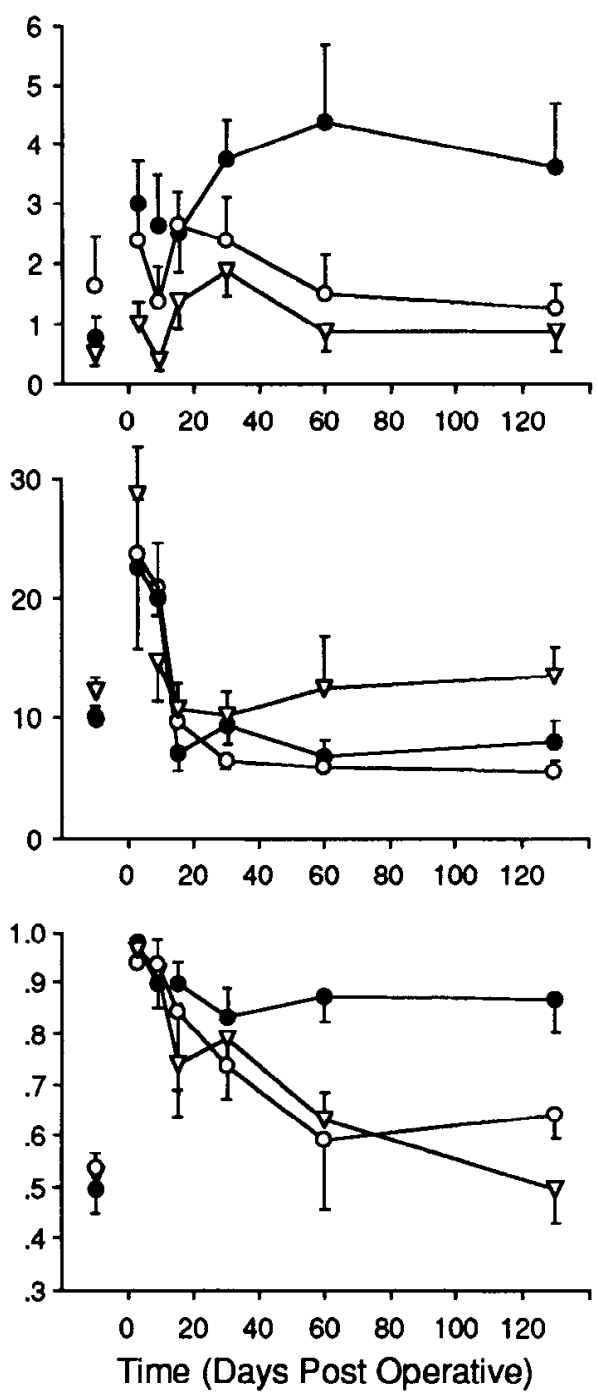

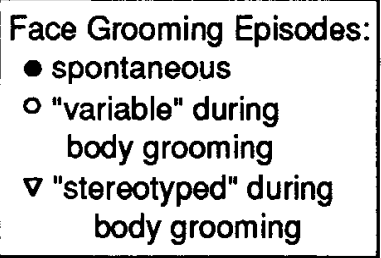

Face Grooming Episodes:

- spontaneous

"variable" during

body grooming

body grooming

Figure 5. Comparisons of three types of face-grooming episodes. $A-C$, Line graphs representing frequency $(A)$, duration $(B)$, and degree of asymmetry (the proportion of unilateral medium face-wash strokes directed to the territory of the affected IoN) $(C)$, of face-grooming episodes categorized as spontaneous (solid circles), "variable" during body grooming (open circles) and "stereotyped" during body grooming (triangles), observed in shamoperated rats (left) and in IoN ligation rats (right), before the operation (Pre) and at six postoperative time points. Plotted data are mean values. Error bars indicate the SEMs. Before the operation, no differences were found between the three types of face-grooming episodes within or between the groups for any of the three measures [RM-ANOVA: episodes, all $F(2,28) \leq 1.363, p \geq 0.2719$; episodes $\times$ group, all $F(2,28) \leq 0.007, p \geq$ 0.9929].

the ligated nerve-characterized all types of face grooming (spontaneous, "variable" during body grooming and "stereotyped" during body grooming) or only a specific type. Figure $5 \mathrm{~A}$ illustrates that the increase in the number of face-grooming episodes that occurs after day 15 in IoN ligation rats (see Fig. 2) was found only for spontaneous episodes, whereas the frequency of the other two types of episodes showed a concomitant decrease [RM-ANOVA: episodes $\times$ group, $F(2,28)=4.222, p$ $<0.05$; episodes $\times$ time postoperative $\times$ group, $F(10,140)=$ $1.951, p<0.05$ ]. Figure $5 B$ illustrates that the initial increase and subsequent decrease in episode duration were characteristic of all three types of episodes observed in IoN ligation rats [RMANOVA: time postoperative $\times$ group, $F(5,70)=8.348, p<$ 0.0001 ; episodes $\times$ time postoperative $\times$ group, $F(10,140)=$ 0.986 , NS]. Figure $5 C$ illustrates that all three types of episodes were initially characterized by a highly asymmetric distribution of unilateral medium-sized face-wash strokes immediately following IoN ligation [RM-ANOVA: time postoperative $\times$ group, $F(5,70)=4.813, p<0.001$; episodes $\times$ group, $F(2,28)=10.271$, $p<0.002]$; however, this asymmetry persisted only for spontaneous episodes, whereas the distribution observed in the other two types of episodes gradually returned to the approximately symmetrical distribution found preoperatively [RM-ANOVA: episodes $\times$ time postoperative $\times$ group, $F(10,140)=2.117, p$ $<0.05]$.

\section{Thigmotactic scanning}

No differences between IoN ligation rats or sham-operated rats could be found for (1) the amount of thigmotaclic scanning activity [overall mean: IoN, $18.3 \pm 10.2 \mathrm{sec}$; sham, $16.6 \pm 12.2$ sec; $F(1,14)=0.326$, NS] or (2) the proportion of thigmotactic scanning activity performed with the vibrissae ipsilateral to the 
experimental surgery [overall mean: IoN, $0.43 \pm 0.28$; sham, $0.51 \pm 0.27 ; F(1,14)=2.541, \mathrm{NS}]$. Only on the first 2 postoperative days of testing ( 3 and 6 ), the proportion of thigmotactic scanning performed with the ipsilateral vibrissae was significantly lower than preoperatively in IoN ligation rats [post hoc $t$ tests, respectively: $t(7)=4.332, p<0.01 ; t(7)=3.012, p$ $<0.05]$.

\section{Body weight}

IoN ligation rats had a lower average daily weight gain $(2.7 \pm$ $0.3 \mathrm{gm} / \mathrm{d})$ than sham-operated animals $(3.4 \pm 0.5 \mathrm{gm} / \mathrm{d})$ [unpaired $t$ test, $t(14)=2.836, p<0.05$ ]. This finding was confirmed by the significantly lower end-weight (on postoperative day 130) of IoN ligation rats $(652.4 \pm 51.3 \mathrm{gm})$ compared to shamoperated rats $(717.6 \pm 73.2 \mathrm{gm}$ ) (unpaired $t$ test, $t(14)=2.657$, $p<0.05$ ]

\section{Additional observations}

Ipsilateral medium face-wash strokes observed in IoN ligation rats often appeared in uninterrupted sequences of three or four strokes (maximum observed uninterrupted sequence, 13 strokes). Preoperatively or in sham-operated rats ipsilateral medium facewash strokes appeared in pairs or as single strokes. In all IoN ligation animals, the prolonged presence (up to 2 months following the operation) of chromorrhea (red tears) was observed ipsilateral to the ligation. Chromorrhea was also observed in sham-operated rats but only on $3 \mathrm{~d}$ postoperative. From the first day after the operation, large droplets of saliva were observed in the comer of the mouth of all IoN ligation rats. This hypersalivation lasted throughout the study and was never observed in sham-operated animals. From postoperative day 25 all IoN ligation rats had distinct scratch marks within the IoN territory ipsilateral to the ligation. On postoperative day 40 , three IoN ligation rats showed wounds in the neck area. Two other IoN ligation rats showed bilateral scratch marks on day 130 postoperatively. Scratch marks were never observed in shamoperated animals. During postoperative observation sessions, IoN ligation rats had a significantly higher defecation rate than sham-operated rats [RM-ANOVA, $F(1,14)=10.215, p<0.005$ ]. Unoperated rats and sham-operated rats excreted an average of 2.1 boli during the $7 \mathrm{~min}$ observation period, while IoN ligation rats excreted between four and eight boli (mean over all postoperative time points, 4.7 ).

\section{Responses evoked by mechanical facial stimulation}

\section{Responses in preoperative and sham-operated rats}

Responses to mechanical stimulation were scored based on the number of observed response elements that were assumed to reflect the aversiveness of the evoked sensation. A strong positive correlation was found between response scores and stimulus intensity in preoperative rats (Fig. 8), which supports the validity of the scores. In sham-operated rats, posioperative responses did not differ from preoperative responses [Friedman ANOVA, one for each area and each intensity, all $\chi(13)<$ 22.362, NS] (Figs. 6, 7), nor were responses to stimulation of the ipsilateral IoN territory different from responses to stimulation outside this area [Friedman ANOVA, one for each time point and each intensity, all $\chi(3)<7.3, \mathrm{NS}]$. As a result, the positive stimulus-response relations observed preoperatively remained unchanged for each of the areas (Fig. 8; see caption for stimulus-response correlations). The latter finding addresses the concern that responses to repeated applications of the same series of mechanical stimuli might be affected by learning, fatigue, increased irritability, or other nonspecific factors.

\section{Changes in responses following IoN ligation}

Ligation of the IoN produced dramatic changes in the responses of rats to mechanical stimulation of the territory of the ligated nerve [Friedman ANOVA, all $\chi^{2}(13)>40.081, p<0.0001$ ] (Figs. 6,7). The changes consisted of an initial decrease (on postoperative days 3 and 6) and a subsequent increase (around postoperative 12) in the number of evoked response elements (Fig. 6A). Some IoN ligation rats responded more intensely from the first postoperative day. However the majority of rats showed a "no response" on postoperative days 3 and 6 , especially with lower intensities ( $1 \mathrm{gm}, 2 \mathrm{gm}$, and $4 \mathrm{gm}$ ) (Fig. $8 \mathrm{~A}$ ). In some IoN ligation rats, higher intensities $(9 \mathrm{gm}, 16 \mathrm{gm}$, and especially pin prick) applied to the ligated IoN territory evoked responses similar to those observed preoperatively (Figs. 6, 8A). Due to this apparent variability in evoked responses within the IoN ligation group during the first postoperative sessions, no significant differences with preoperative responses were found on postoperative days 3,6 , and 9 (post hoc comparisons, NS; see Fig. 7). From postoperative day 12, stimulation of the territory of the ligated nerve evoked consistently the most aversive response categories (strong aversive response and prolonged aversive response), and this for each of the stimulus intensities (Figs. $6 A, 8 A$ ). From postoperative day 12 , evoked responses were found to be significantly greater than the preoperative responses. Responses to lower intensities (1 gm and $2 \mathrm{gm}$ ) remained significantly greater than preoperative responses up to postoperative day 80 . Responses to the most intense stimuli failed to be significantly different from preoperative levels beyond postoperative day 50 (16 gm and pin prick) and day 60 (4 gm and 9 gm) (post hoc comparisons, $p<0.05$; see Fig. $7 A$ for details).

Responses to stimulation of facial areas outside the territory of the ligated nerve also changed significantly over time, but only for lower stimulus intensities and never for $16 \mathrm{gm}$ or pin prick stimulation [contralateral IoN territory: $1 \mathrm{gm}, 2 \mathrm{gm}, 4 \mathrm{gm}$, $9 \mathrm{gm}$; Friedman ANOVA, all $\chi^{2}(13)>32.824, p<0.005$; outside IoN territory ipsilateral and outside IoN territory contralateral: $1 \mathrm{gm}, 2 \mathrm{gm}, 4 \mathrm{gm}$; Friedman ANOVA, all $\chi^{2}(13)>$ 28.625, $p<0.01$ ] (Fig. $6 B-D$ ).

Although the changes in responsiveness to stimulation of facial areas outside the territory of the ligated nerve appeared to have a similar time course (Fig. 6), post hoc comparisons with preoperative responses indicated that increases in responses to stimulation of the contralateral IoN territory reached statistical significance earlier (around postoperative day 15), than those to stimulation of areas outside the IoN territory, ipsi- and contralateral to the operation (around postoperative days 30-40) [post hoc comparisons, $p<0.05$ ] (see Fig. $7 B-D$ for details). None of the changes in responses to stimulation of facial areas outside the ligated IoN territory lasted longer than postoperative day 80 .

In spite of the significant changes in responsiveness to stimulation of areas outside the ligated IoN territory, the "highest" response category, "prolonged aversive behavior" (consisting of a withdrawal reaction, escape/attack behavior, and typified by a bout of intense grooming directed to the stimulated area), was observed almost exclusively in response to stimulation of the ligated IoN territory. The highest level of response to stimulation of other facial areas was intense escape or attack behavior. The few "prolonged aversive behavior" responses to stimula- 
Figure 6. Time course of changes in responses evoked by mechanical stimulation of the face of sham-operated rats and rats with an IoN ligation. $A-D$, Line graphs representing the mean response scores of sham-operated rats (left $\mathrm{col}$ $u m n$ ) and IoN ligation rats (right column) to von Frey hair stimuli of $1 \mathrm{gm}$ (solid circles), 2 gm (open inverted triangles), $4 \mathrm{gm}$ (solid inverted triangles), $9 \mathrm{gm}$ (open squares), and $16 \mathrm{gm}$ (solid squares), and pin prick stimulation (open triangles), applied to each of the four stimulated facial areas $(A-D)$, before the operation (Pre) and at postoperative time points. See Results for statistics.

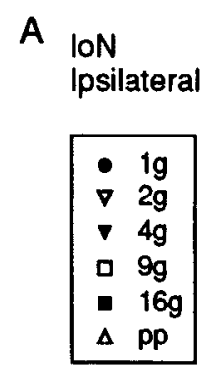

B ION

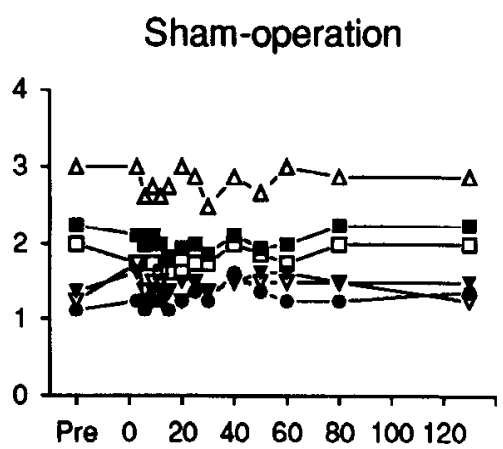

$\begin{array}{llllllll}\text { Pre } & 0 & 20 & 40 & 60 & 80 & 100 & 120\end{array}$

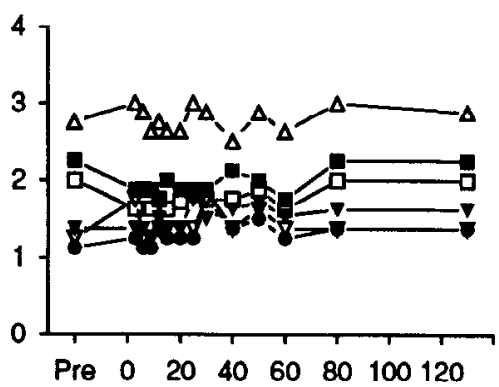

C Outside loN Ipsilateral

\section{4}

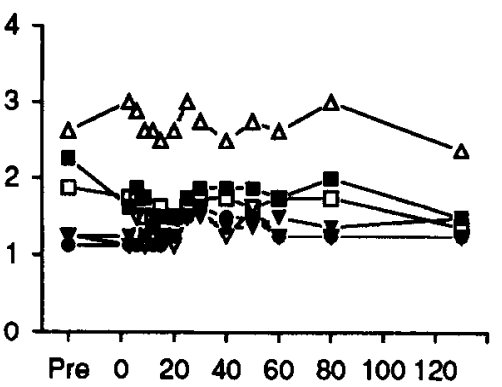

D Outside loN Contralateral

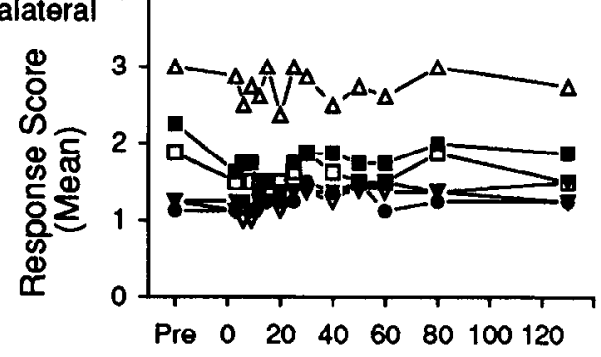

Time (Days Post Operative)
IoN Ligation
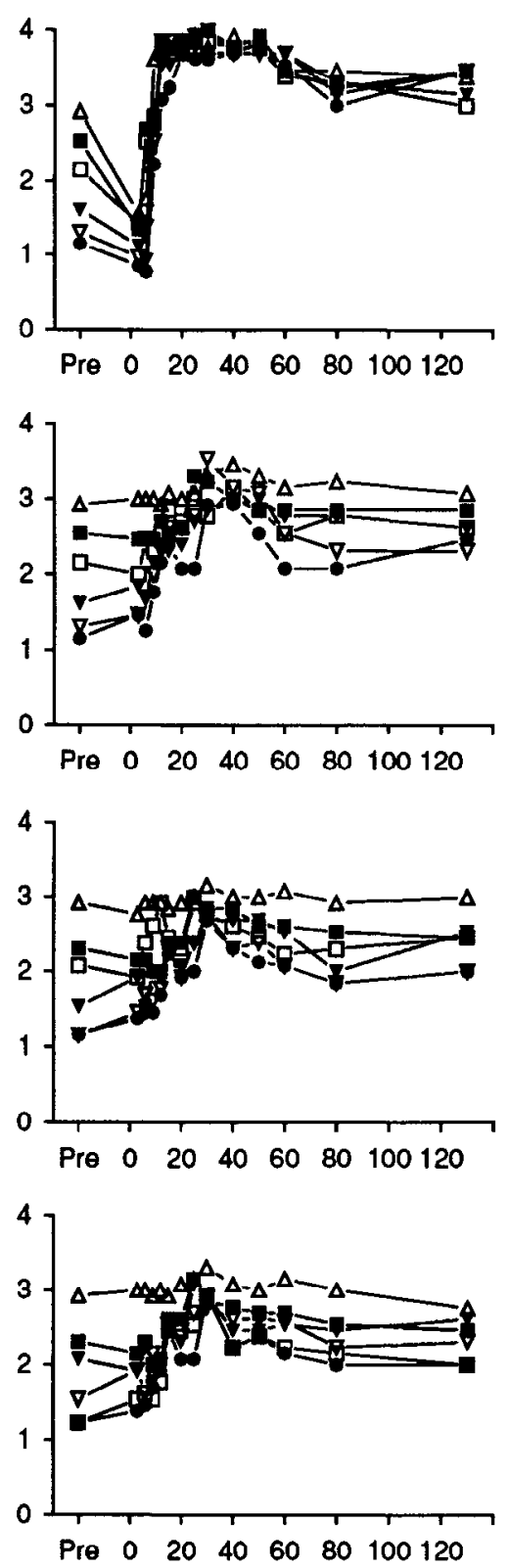

tion of areas outside the ligated IoN territory were all observed in response to stimulation of the contralateral IoN territory (Fig. 6).

Preoperative, "early" postoperative (3-12 d) and "late" postoperative (15-130 d) stimulus-response curves (Fig. 8) can be used to estimate the specific alterations in facial somatosensory function that are likely on the basis of observed changes in responsiveness of IoN ligation rats (see Fig. 8 caption for estimated stimulus-response correlations). These graphs show that the IoN territory is the only facial area that is hypoesthetic in the early postoperative period and profoundly allodynic/hyperalgesic in the latc postoperative period (Fig. 8A). The graphed stimulus-response curves also indicate some degree of hypersensitivity for the other facial areas of IoN ligation rats in the late postoperative period (Fig. $8 B-D$ ).

\section{Difference scores}

Difference scores were calculated to provide a general measure of changes in responsivity to stimulation per facial area and over stimulus intensities (Fig. 9). For cach of the facial areas, median difference scores of sham-operated rats were close to 0 at all postoperative time points. IoN ligation rats showed biphasic alterations of the difference scores for the ligated IoN territory: on postoperative days 3 and 6 difference scores of IoN ligation rats were significantly lower compared to sham-operated rats [Mann-Whitney $U$ tests, both $Z(U) \leq-2.065, p \leq$ 0.05 ]; from postoperative day 12 to the end of the study, IoN ligation rats had significantly higher difference scores [MannWhitney $U$ tests, all $Z(U) \leq-3.215, p \leq 0.002]$. For facial areas outside the ligated IoN territory, significantly higher dif- 
ference scores than in sham-operated rats were present from postoperative day 15 up to postoperative day 60 [Mann-Whitney $U$ tests, all $Z(U) \leq-1.963, p<0.05$ ]

Difference scores for both the ipsi- and contralateral IoN territory remained significantly different from those observed in sham-operated rats after postoperative day 60 (Fig. 9A-D). The graphical representation of the difference scores shows (1) that the territory of the ligated IoN is the only facial region in which hypoesthesia was present early after the injury, (2) that in the late postoperative period, a greater degree of hypersensitivity was present in the ligated IoN territory, and (3) that maximal changes in responsiveness occurred around the same postoperative time (postoperative days 15-40).

\section{Time course of changes in nonevoked and evoked behavior following ioN ligation}

Changes in both nonevoked and evoked behavior after IoN ligation followed a time course that may be divided into an early period (1-12 d postoperative) and a late period (15-130 d postoperative). In the early period immediately following the $\mathrm{CCI}$, there was a dramatic increase in face grooming directed specifically to the territory of the ligated IoN that appeared concurrently with a decreased responsiveness to mechanical stimulation within this territory. The increased face-grooming activity that occurred in this early period consisted of an increase in both the frequency and the duration of face-grooming episodes.

Between postoperative days 12 and 15 , the hyporesponsiveness of the ligated IoN territory to mechanical stimulation reversed and was replaced by a dramatic hyperresponsivity, which appeared as a maximal response (prolonged aversive behavior) to both high and low intensities of mechanical stimulation. Concurrent with this increased responsivity, the frequency of (nonevoked) face-grooming episodes remained elevated while their duration, which was initially elevated as well, decreased sharply to become significantly shorter than preoperatively.

\section{Discussion}

\section{CCI-induced behavioral changes}

The results of the present study show that CCI to the IoN leads to quantitative changes in free, nonevoked behavioral activity and in responses evoked by mechanical stimulation of the face. Quantified behavioral changes develop in two phases. In an early postoperative period (1-12 d postoperative), changes in nonevoked behavior are maximal: rats groom the territory of the injured nerve very frequently and for long periods; they show a profound decrease in exploratory behavior and spend most of the observation time in an immobile posture. During the early period after IoN ligation, rats do not respond to low-intensity stimulation of the ligated nerve territory; intense stimulation evokes in some rats responses similar to preoperative responses. For some IoN ligation rats the period of rclative hyporesponsiveness is shorter than 12 postoperative days. In the late postoperative period (from postoperative day 15) the prevalence of asymmetric face-grooming behavior observed in IoN ligation rats is markedly reduced. However, the total time spent face grooming remains significantly higher than in control rats: after postoperative day 15 , IoN ligation rats perform more facegrooming episodes of a shorter duration. The asymmetric distribution of face-wash strokes that was initially present in all types of face-grooming episodes persists beyond the early postoperative period in spontaneous episodes only. From postop-
Time (Days Post Operative)

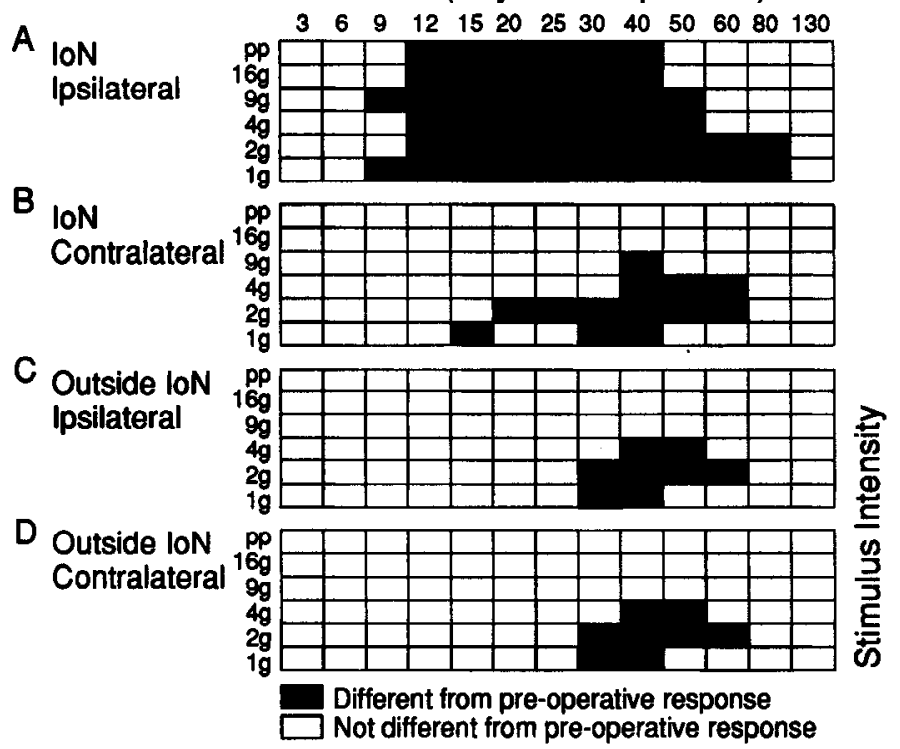

Figure 7. Schematic overview of the extent and duration of changes in responses to mechanical stimulation following an IoN ligation. $A-D$, Solid cells indicate on which postoperative days $(x$-axis $)$ and for which stimulus intensities ( $y$-axis) responses of IoN ligation rats to stimulation of each of the four stimulated facial areas $(A-D)$ were significantly different from the preoperative responses. To determine significance levels, post hoc within-group comparisons were done per stimulus intensity and per stimulated area following a Friedman ANOVA for the factor "time postoperative." Comparisons were based on the ranking of the mean response scores obtained for each test point.

erative day 15 , IoN ligation rats become hyperresponsive to all stimulus intensities applied to the ligated IoN territory. Concurrent with the hyperresponsiveness to stimulation of the ligated IoN territory, IoN ligation rats show a limited increase in the responsiveness to stimulation of the IoN territory contralateral to the ligation; 2-3 weeks after the onset of the hyperresponsiveness to stimulation of the ligated IoN territory, responsiveness to stimulation of facial areas outside the affected area is also increased.

\section{CCI-induced changes in somatosensory function}

Mechanical allodynia. The complete loss of the stimulus-response relation for the territory of the ligated nerve, with all stimulus intensities evoking the maximal response, strongly suggests the presence of major sensory disturbances in this facial area. Although sensory thresholds were not tested in this study, it appeared that after the ligation both pain and detection thresholds (defined as the lowest stimulus intensities that evoked, respectively, a strong aversive response and a mild aversive response) initially showed a substantial increase, followed by a dramatic decrease. The decrease in pain and detection thresholds was accompanied by an amplification of the afferent signal as evidenced by the maximal response to even the weakest stimulus intensities.

The evoked responses are almost identical to those evoked by stroking the face of cats (King et al., 1956; King and Barnett, 1957; King, 1970; Black, 1974) or rats (Kryzhanovsky et al., 1974; Sakai et al., 1979) with an experimentally created epileptic focus in the trigeminal nucleus caudalis. This hyperresponsiveness was also observed in cats following insertion of chromic gut sutures in the vicinity of the trigeminal root (Burchiel, 1980). The hyperresponsiveness found in rats with a CCI to the IoN 
Pre Operative

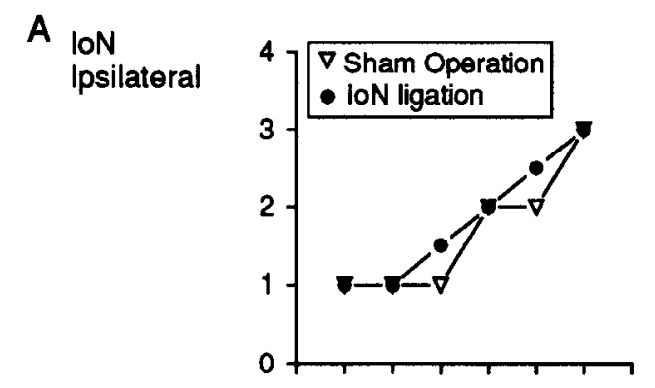

B ION loN

Outside
Ipsilateral

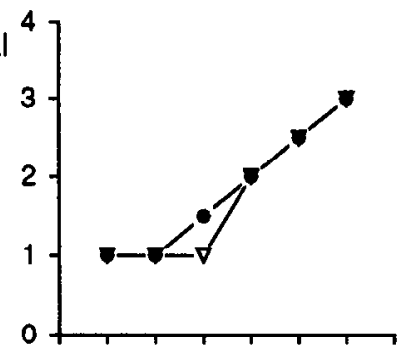

$1 g \quad 2 \quad 4 g \quad 9$ 16g pp

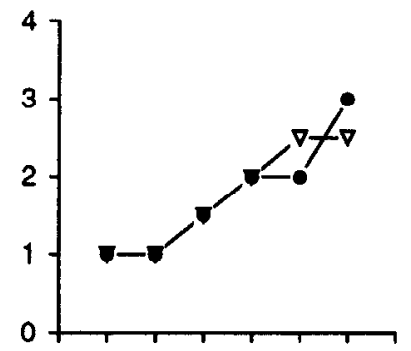

$1 g \quad 2 g \quad 4 g \quad 9 g 16 g$ pp

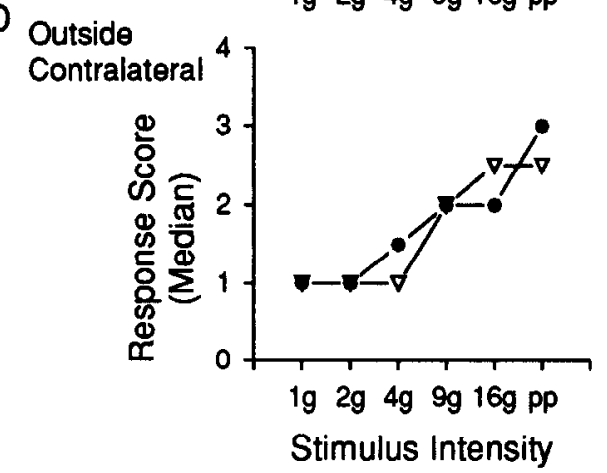

Early PO Period

Late PO Period

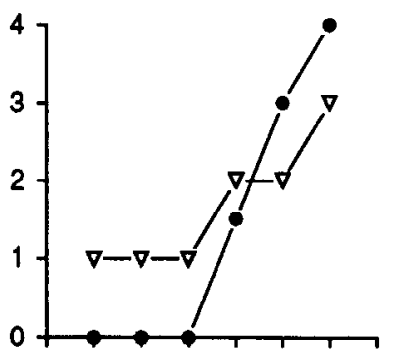

$1 \mathrm{~g} 2 \mathrm{~g} 4 \mathrm{~g} 9 \mathrm{~g} 16 \mathrm{~g} p \mathrm{p}$

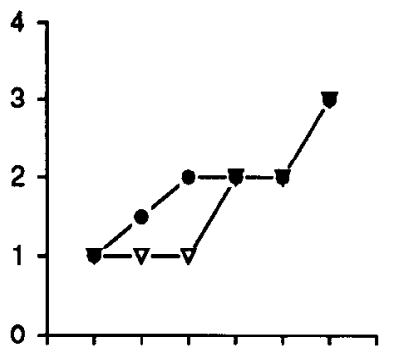

1g $2 g \quad 4 g$ g $16 g$ pp

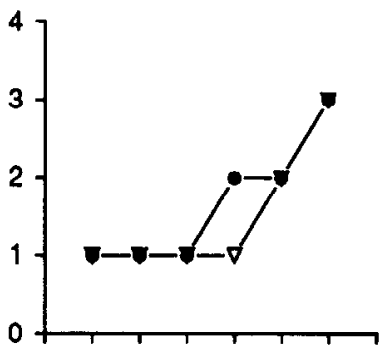

$1 \mathrm{~g} 2 \mathrm{~g} 4 \mathrm{~g} 9 \mathrm{~g} 16 \mathrm{~g} \mathrm{pp}$

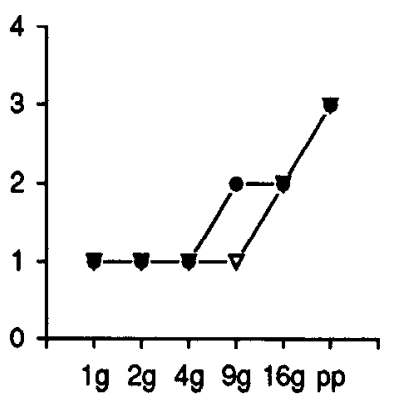

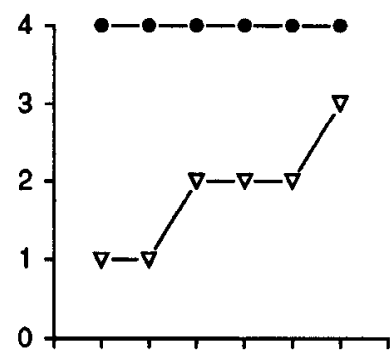

$1 \mathrm{~g} 2 \mathrm{~g} 4 \mathrm{~g} 9 \mathrm{~g} 16 \mathrm{~g} \mathrm{pp}$

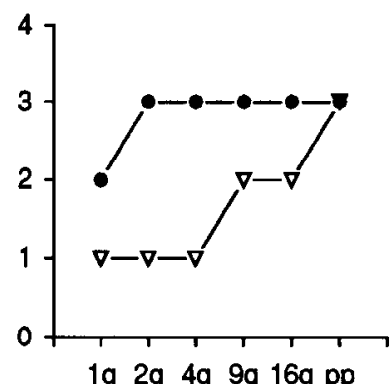

$1 \mathrm{~g} 2 \mathrm{~g} \quad 4 \mathrm{~g} 9 \mathrm{~g} 16 \mathrm{~g} p \mathrm{p}$

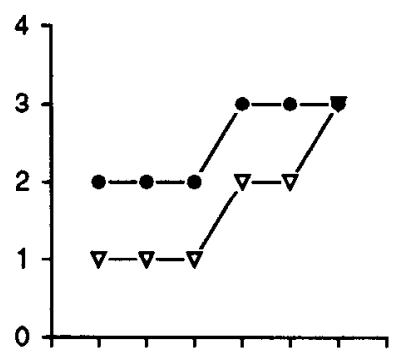

$1 \mathrm{~g} \quad 2 \mathrm{~g} \quad \mathrm{gg} 9 \mathrm{~g} 16 \mathrm{~g} p \mathrm{p}$

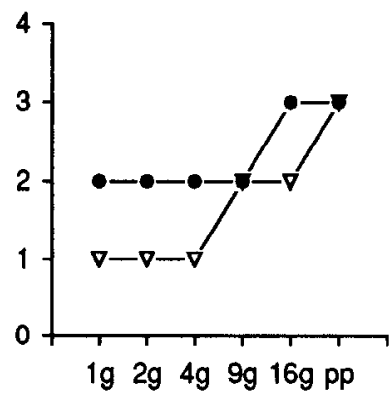

Figure 8. Postoperative changes in stimulus-response relation. $A-D$, Line graphs representing the median response scores of sham-operated rats (triangles) and loN ligation rats (circles), evoked by each of the stimulus intensities ( $x$-axis), before the operation (Pre Operative), from 3 to $12 \mathrm{~d}$ postoperative (Early PO Period), and from 15 to 130 d postoperative (Late PO Period), for each of the four stimulated facial areas ( $A-D$ ). Postoperative medians of each group were based on the average response score, over 13 time points, per rat. Preoperatively, Goodman-Kruskal $G$ coefficients were between 0.77 and 0.79 in both groups for each of the stimulated areas. In the "early PO period," $G$ coefficients were $(A-D$, respectively) 0.57 , $0.65,0.62$, and 0.64 in sham-operated rats, and $0.12,0.48,0.56$, and 0.55 in IoN ligation rats; in the "late PO period" averaged (see Materials and Methods) $G$ coefficients were $(A-D$, respectively) $0.59,0.62,0.61$, and 0.51 in sham-operated rats, and $-0.02,0.27,0.41$, and 0.42 in IoN ligation rats.

also resembles the so-called "touch-evoked agitation" observed in rats following intrathecal administration of strychnine or bicuculline at the level of the spinal dorsal horn (Yaksh, 1989).

The observed behavioral abnormalities are reminiscent of the psychophysical phenomenon of "mechanical allodynia," a type of somatosensory abnormality often found in neuropathic pain patients (Lindblom and Hannson, 1991). The term "mechanical allodynia" describes "abnormal" pain sensations elicited by in- nocuous mechanical stimuli that, when evoked, radiate far beyond the point of stimulation and outlast the stimulus for several seconds (Lindblom and Hannson, 1991). Trigeminal neuralgia, also called "tic douloureux" (André, 1756; Fromm and Sessle, 1991), is typified by an extreme form of mechanical allodynia: patients suffer recurrent attacks of facial pain of an extreme intensity, often experienced as explosive and electric shock-like sensations. These abnormal pain sensations are most effectively 
elicited by innocuous vibratory and weak tactile stimuli ( $\mathrm{Ku}$ gelberg and Lindblom, 1959). Allodynia, as defined by Lindblom and Hannson (1991), appears more like an all-or-none phenomenon than a graded function of the stimulus intensity such as hyperalgesia (in which pain intensity increases with stimulus strength, but with a steeper stimulus-response function than normal; Lindblom and Hannson, 1991). The changes in the stimulus-response relation observed after $\mathrm{CCI}$ to the IoN conform to this definition of allodynia.

The increased responsiveness to stimulation of facial areas outside the ipsi- or contralateral IoN territory, which starts about 2-3 weeks later, is less likely to be caused by the presence of specific somatosensory changes in the respective areas. The hyperresponsiveness follows the same time course and is practically equal for the two sites. In contrast to the ligated IoN territory, almost none of the stimulations is capable of evoking the "highest" response category in these areas. Responses to these areas are typified by the presence of intense avoidance behavior. It is therefore more likely that they are caused by increased levels of stress and anxiety in IoN ligation rats resulting from the specific (probably painful) somatosensory changes in the affected region.

The increased responsiveness to stimulation of the IoN territory contralateral to the ligation develops simultaneously with the hypersensifivity of the ligated IoN territory, and is less intense compared to the affected area but more intense and lasts longer compared to the areas outside the IoN territory. The few "prolonged aversive behavior" responses to stimulation of areas outside the ligated IoN territory are all observed in response to stimulation of the contralateral IoN territory. Changes in responses to stimulation of the contralateral IoN territory may therefore result from increased somatosensitivity rather than from a higher stress level alone.

Paresthesias/dysesthesias. The appearance of asymmetric facegrooming episodes with face-wash strokes directed to the territory of the ligated IoN represent most likely a behavioral manifestation of spontaneous, somatic sensations in this region. The phenomenologic similarity between abnormal face-grooming activity observed after IoN ligation and face grooming of normal rats in response to subcutaneous formalin (Clavelou et al., 1989; Klein et al., 1991; Eisenberg et al., 1993) or noxious heat or noxious mechanical stimulation (Rosenfeld et al., 1978; Morris et al., 1982; Cahusac et al., 1990) applied to the IoN territory suggests that asymmetric face grooming is a sign of facial pain. Observed face-grooming patterns were also analogous to those observed in rats following chemical irritation of the trigeminal nucleus caudalis (Kryzhanovsky, 1974; Sakai et al., 1979) or following placement of chromic gut sutures under the trigeminal root in cats (Burchiel, 1980).

In spite of the phenomenologic similarities between asymmetric face-grooming episodes observed after IoN ligation and responses of normal rats to painful facial stimulation, other distortions of sensory input, such as complete or partial numbness (an- or hypoesthesia) or the presence of nonaversive cutaneous sensations (paresthesias, e.g., tingling, prickling) need to be considered likely triggers of asymmetric face grooming as well.

Denervation studies have provided evidence that sensory loss can result in asymmetric face grooming. Asymmetric facegrooming episodes have been observed following unilateral trigeminal rhizotomy in cats (Anderson et al., 1971) and complete unilateral trigeminal denervation in rats (Berridge and Fentress,

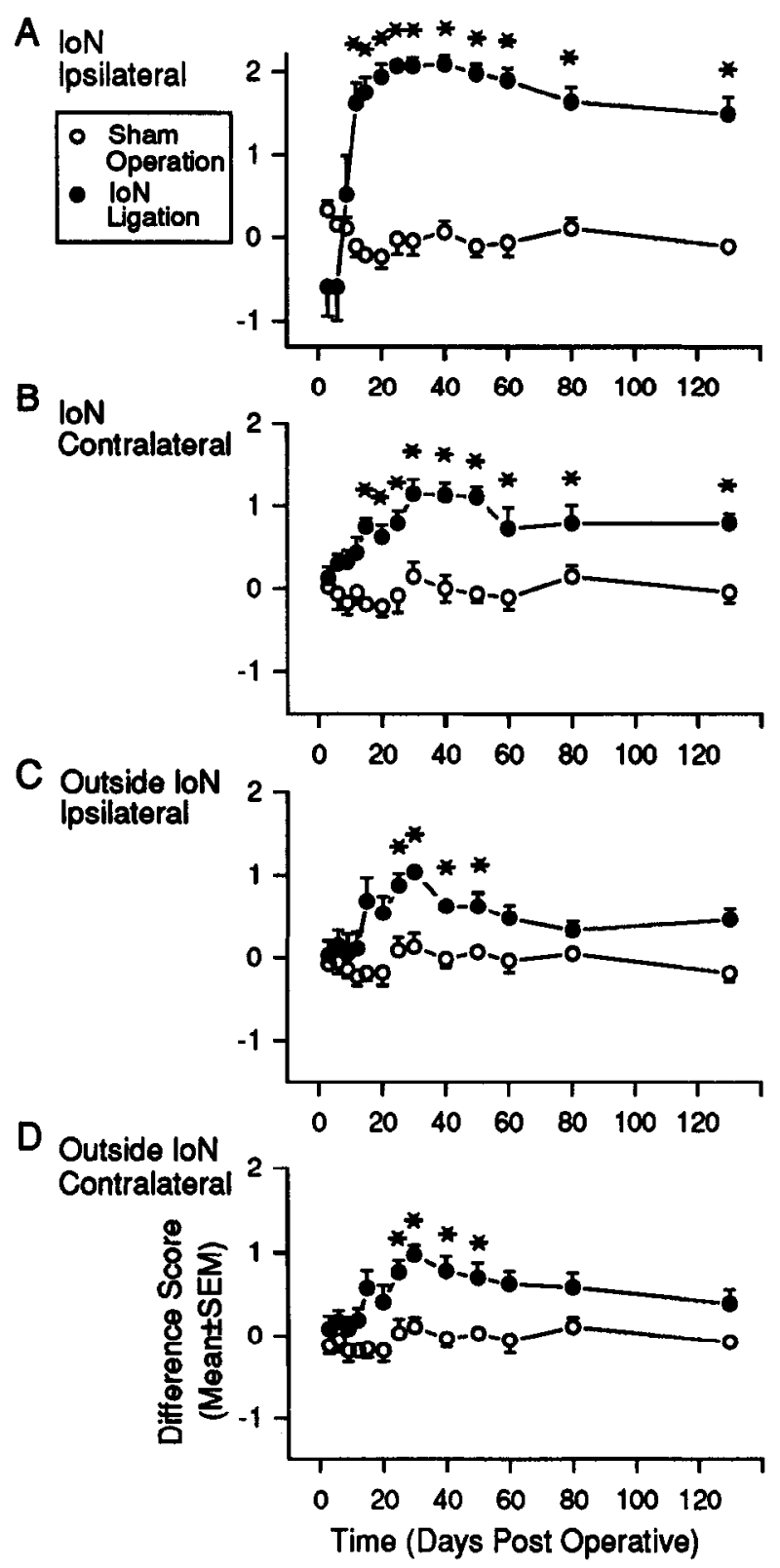

Figure 9. Difference scores. $A-D$, Line graphs representing the mean difference scores (see Materials and Methods) of sham-operated rats (open circles) and IoN ligation rats (solid circles) at 13 postoperative time points for each of the four stimulated facial areas $(A-D)$. Error bars indicate SEM. Significant differences between groups, as determined by post hoc comparisons, are indicated by asterisks $\left({ }^{*}, p<0.05\right)$.

1986). On the other hand, some evidence exists that acute anesthetic blockade of the IoN with lidocaine does not evoke asymmetric face grooming directed to the affected IoN territory (B. P. Vos, A. M. Strassman, and R. J. Maciewicz, unpublished observations). Another argument against anesthesia as trigger for asymmetric face grooming following IoN ligation is the fact that asymmetric face-grooming episodes persist after all signs of hypo-(or an)esthesia have disappeared.

Nonaversive sensations such as innocuous tingles or pricks, or strange, nonfamiliar sensations due to impaired cutaneous and vibrissal function, are other possible triggers of asymmetric face grooming. Considering the importance of the vibrissae as a sense organ for rats (Vincent, 1913), a unilateral distortion 
(either a decrease or a transformation) of the vibrissal input, normally evoked by continuous whisking motions during scanning activity, can constitute an important abnormal sensory condition for IoN ligation rats. The lack of long-term disturbances of thigmotactic scanning behavior suggest that vibrissal function was intact. However, more sophisticated tests of vibrissal discriminatory function (e.g., Carvell and Simons, 1990; Tomie and Whishaw, 1990) might reveal alterations in function that were not detected in the present study.

Ethologic studies in rodents have demonstrated that mild irritation of restricted areas of the pelage elicits transitory grooming directed to the irritated area (e.g., Griswold et al., 1977). However, procedures such as application of mineral oil to the vibrissal pad or unilateral vibrissae clipping fail to evoke persistent asymmetric face grooming directed to the affected IoN territory (Vos, Strassman, and Maciewicz, unpublished observations). Continuous presence of a foreign substance on one side of the face might, indeed, evoke recurrent episodes of asymmetric face grooming with face-wash strokes directed to that side, although the continuous presence of a foreign body is assumed to produce aversive rather than nonaversive, nonpainful sensations.

In light of the hypersensitivity of the ligated IoN territory to mechanical stimulation, the increased face grooming directed to this area seems to be a paradoxical response that would, itself, be expected to produce painful sensations. There are, in fact, some signs that this behavior is negatively reinforced, beginning at the time of the onset of mechanical allodynia; these include a decrease in the duration of all types of face-grooming episodes, and a decrease in the asymmetry of face-grooming episodes associated with body grooming. However, the negative reinforcement is apparently not sufficient to produce complete extinction of the pain-producing face-grooming activity, and in fact the frequency of spontaneous face-grooming episodes continues to increase at this time. This apparent paradox, of the persistencc of asymmetric face grooming directed to the ligated IoN territory after the development of mechanical allodynia, might be explained if the sensations that triggered the abnormal grooming were more intensely aversive than the allodynic sensations that result from the grooming. If the asymmetric face grooming were triggered by nonaversive sensations, it might not be expected to persist following the development of allodynia.

Despite these arguments, it remains plausible that hypo- or anesthesia or nonaversive paresthesias are, in part, triggering asymmetric face grooming during the first postoperative week. However, IoN ligation rats not only showed specific alterations in face-grooming behavior; they also exhibited typical nonspecific behavioral signs of discomfort and stress commonly found in animals in a persistent pain condition (O'Kelly and Steckle, 1939; Grey and Lalljee, 1974; Morton and Griffiths, 1985; National Research Council, 1992). These signs (including slower body weight gain, less exploratory behavior, more freezing-like behavior, a higher defecation rate, and chromorrhea) were also maximal during the early postoperative period.

Time course. Based on this discussion, somatosensory changes following IoN ligation can be divided into (1) an early period, immediately after the $\mathrm{CCI}$, during which the ligated IoN territory is hypo- or anesthetic, and (2) a late postoperative period, starting after the second postoperative week, during which the territory of the ligated IoN becomes hypersensitive. The abnormal, asymmetric face-grooming activity displayed by IoN ligation rats is maximal in the early postoperative period and weakens in the later period, once hypersensitivity of the ligated IoN territory is present. It is hypothesized that the asymmetric face-grooming episodes might constitute responses to intense, irritative, or painful sensations that occur spontaneously or that are triggered by various types of self-initiated facial stimulation not under the control of the experimenter. The fact that asymmetric face grooming decreases in the late period could be explained by the presence of hypersensitivity in this region.

\section{Comparison with other rat models of neuropathic pain}

Rats with a CCI to the sciatic nerve (Bennett and Xie, 1988; Seltzer et al., 1990; Kim and Chung, 1992) develop signs of hypersensitivity much earlier (around postoperative day 3 ) than IoN ligation rats. The later development of hypersensitivity could be related to the slightly modified constriction procedure: only two instead of four sutures of a smaller gauge (5-0 instead of 4-0) were used. In addition, only changes in mechanosensitivity were studied, so earlier thermal hypersensitivity, if present, remained undetected. Early mechanical hypalgesia has also been observed after CCI to the sciatic nerve (Attal et al., 1990; Ro and Jacobs, 1993) but for a substantially shorter postinjury period (maximal $4 \mathrm{~d}$ ). The prolonged hypalgesia could be related to a possibly higher degree of nerve constriction due to pressure of adjacent bony structures against the ligatures around the IoN. Observed changes in mechanosensitivity after CCI to the IoN followed an identical time course as behavioral signs of altered thermal sensitivity following CCI to the sciatic nerve in rats with a transected saphenous nerve (Ro and Jacobs, 1993). The specific structure of the trigeminal system may prohibit injurycompensating actions of adjacent nerves.

Observed asymmetric face grooming can be considered equivalent to bouts of grooming (licking and biting movements) directed to the ipsilateral hind paw following complete or partial sciatic nerve ligation in Sprague-Dawley rats (Bennett and Xie, 1988; Seltzer et al., 1990; Kim and Chung, 1992). Grooming actions directed to the ipsilatcral hind paw may constitute a behavioral manifestation of spontaneous pain in the territory of the ligated sciatic nerve although the incidence and duration of this behavior has not been quantified. The time course of alterations in face-grooming behavior displayed by SpragueDawley rats with an IoN ligation is comparable to the time course of ipsilateral "scratching behavior" displayed by Wistar rats with a CCI to the sciatic nerve (Kupers et al., 1992). This "scratching activity" was used as an indication of spontaneous pain since it resembles shaking and scratching movements observed in normal rats in response to intense noxious stimulation of the plantar surface of the foot of normal rats (Sherrington, 1910).

Although CCI to the IoN leads to comparable behavioral changes as $\mathrm{CCI}$ to the sciatic nerve, changes in responses to mechanical stimulation of the territory of the injured nerve do not occur simultaneously but later than behavioral indications of spontaneous pain (i.e., asymmetric face grooming).

\section{Hypothetical neuropathologic mechanisms}

The initial period of impaired mechanosensitivity is most likely related to a substantial degeneration of myelinated axons that is observed following a CCI of a peripheral nerve (Gautron et al., 1990; Basbaum et al., 1991; Carlton et al., 1991; Kajander and Bennett, 1992; Munger et al., 1992). Perseverance of responses to high intensity mechanostimulation of the ligated IoN territory is consistent with the higher estimated survival rates 
of small myelinated $\mathrm{A} \delta$ and unmyelinated C-fibers (Basbaum et al., 1991; Kajander and Bennett, 1992). Although no quantitative anatomical or electrophysiological studies of sensory afferent pathology following IoN ligation were performed, electron and light microscopic examination of the ligated IoN early (postoperative day 9) and late (postoperative day 130 ) post-CCI provided a similar picture of axonal pathology as found after CCI to the sciatic nerve (Vos and Maciewicz, 1990; B. L. Munger, personal communication). These observations are partly corroborated by findings that fluoride-resistant acid phosphatase is temporarily depleted early following the $\mathrm{CCI}$ in the ipsilateral superficial medullary dorsal horn while no change in substance P-like immunoreactivity was found in this region of the same animals (Vos and Maciewicz, 1990).

Electrophysiological studies in rats with a CCI to the sciatic nerve have found substantial spontaneous discharge of surviving myelinated primary afferents in the early postoperative period (Kajander and Bennett, 1992; Kajander et al., 1992) and also in C-fibers later postinjury (Xie and Xiao, 1990). If observed episodes of ipsilateral face grooming represent reactions to recurrent paresthetic or dysesthetic sensations, spontaneous discharges in surviving or regenerated $\mathrm{A} \beta$-, $\mathrm{A} \delta$-, and $\mathrm{C}$-fibers are likely to be underlying them. Although the presence of abnormal spontaneous primary afferent activity has not been directly studied following $\mathrm{CCI}$ to the IoN, a significant increase in spontaneous c-fos expression was found in laminae I-IV of the medullary dorsal horn ipsilateral to the ligated IoN (Vos et al., 1991).

The increased responses to mechanical stimulation might be the result of cross-excitation of intact IoN afferent fibers by spontaneous or stimulation-evoked activity in injured neighbors, occurring at the level of the trigeminal ganglion (Devor and Wall, 1990). Via cross-excitation in the trigeminal ganglion, low-intensity mechanical sensory input could arouse discharges in A $\delta$ nociceptors. Such cross-modal excitation could account for the increased responsiveness observed on mechanical stimulation of the ligated IoN territory (Devor, 1989).

The observed hyperresponsiveness observed after $\mathrm{CCI}$ to the IoN could also be the result of "central" pre- and postsynaptic structural, chemical, and physiological changes that developed subsequent to the early injury-induced alterations in peripheral afferent nerve functioning. Pathological structural alterations possibly include a redistribution of the central terminals of regenerating or intact myelinated afferents (Woolf et al., 1992). Myelinated afferent terminals, including those of low-threshold mechanoreceptors, have been observed to sprout into more superficial laminae of the spinal dorsal horn from 2 weeks following sciatic or sural nerve transection (Woolf et al., 1992). Such terminal redistribution could result in activation of nociceptive neurons by innocuous stimulation (Laird and Bennett, 1992, 1993; Palacek et al., 1992), which might contribute to the mechanical allodynia obscrved in the present study.

CCI to the sciatic nerve results in changes of neuropeptide levels in intraspinal terminals of injured afferents (Bennett et al., 1989; Cameron et al., 1991; Wakisaka et al., 1992), and altered levels of receptor binding and protein expression have been observed in the ipsilateral spinal dorsal horn (Aanonsen et al., 1989, 1990; Kajander et al., 1990a,b; Cameron et al., 1991; Draisci et al., 1991; Garrison et al., 1991; Stevens et al., 1991). Evidence of degeneration of small, possibly inhibitory, interneurons was also observed in the dorsal horn following CCI to the sciatic nerve (Sugimoto et al., 1990), possibly as a result of NMDA receptor-mediated excitotoxicity (Yaksh et al., 1992; Yamamoto and Yaksh, 1992). Similar central pre- and postsynaptic alterations might contribute to the altered responsiveness observed after CCI to the IoN.

In addition to changes in peripheral sensory nerve functioning and possible structural and chemical reorganization of dorsal horn circuitry, CCI to a sensory nerve might also induce alterations at the level of supramedullary structures: after CCI to the sciatic nerve functional changes in somatosensory processing have been found at the level of the ventrobasal thalamus (Guilbaud et al., 1990) and the somatosensory cortex (Guilbaud et al., 1992). The significant postoperative changes in responses to mechanostimulation of the contralateral IoN territory do suggest the presence of similar higher level alterations after CCI to the IoN. However, trigeminal sensory afferents have been demonstrated to terminate in the contralateral medullary dorsal horn (Jacquin et al., 1990) so CCI to the IoN could also induce local circuitry alterations in the contralateral medullary dorsal horn resulting in contralateral hypersensitivity.

\section{Summary and conclusions}

This study provides further support that CCI to a rat sensory nerve produces behavioral alterations indicative of neuropathic pain. Applied to the IoN, biphasic changes in evoked and nonevoked behavior were produced. Specific phenomenologic similarities between observed behavioral alterations and reported behavioral signs of facial pain and chronic pain in rats suggest the presence of paresthesias/dysesthesias and mechanical allodynia in the territory of the ligated nerve. The most important finding of this study was that behavioral signs of these distinct classes of neuropathic sensory dysfunctions follow a different time course: behavioral signs of spontaneous, nonevoked aversive sensations (asymmetric face grooming) start immediately after the $\mathrm{CCI}$, whereas the development of mechanical allodynia is delayed and preceded by a period of relative hypoesthesia. In this model it is thereforc possible to examinc hypothetical neuropathological mechanisms underlying dysesthesias/paresthesias separately form neuropathological factors that lead to a faulty restoration of somatosensory function.

\section{References}

Aanonsen LM, Kajander KC, Bennett GJ, Seybold VS (1989) Analysis of ${ }^{125} I$-substance $P$ binding sites in rat spinal cord in an experimental model of peripheral neuropathy. Soc Neurosci Abstr 15:103.

Aanonsen LM, Sloan SI, Kajander KC, Bennett GJ, Seybold VS (1990) Changes in PCP binding sites in rat spinal cord in a chronic constriction injury. Soc Neurosci Abstr 16:1073.

Anderson LS, Black RG, Abraham JA, Ward AA (1971) Neuronal hyperactivity in experimental trigeminal deafferentation. J Neurosurg 35:444-452.

André $P$ (1756) Traité sur les maladics de l'urethrc, pp 323-343. Paris: Delaguette.

Attal N, Jazat F, Kayser V, Guilbaud G (1990) Further evidence for "pain-related" behaviours in a model of unilateral peripheral mononeuropathy. Pain 41:235-251.

Basbaum AI, Gautron M, Jazat F, Mayes M, Guilbaud G (1991) The spectrum of fiber loss in a model of unilateral peripheral mononeuropathy. Pain 41:359-367.

Bennett GJ, Hargreaves KM (1990) Reply to Dr. Hirata and his colleagues. Pain 47:255.

Bennett GJ, Xie YK (1988) A peripheral mononeuropathy in rat that produces disorders of pain sensation like those seen in man. Pain 33: 87-107.

Bennett GJ, Kajander KC, Sahara Y, Iadarola MJ, Sugimoto T (1989) Neurochemical and anatomical changes in the dorsal horn of rats with an experimental painful peripheral neuropathy. In: Processing of sen- 
sory information in the superficial dorsal horn of the spinal cord (Cervero F, Bennett GJ, Headley PM, eds), pp 463-471. New York: Plenum.

Berridge KC (1990) Comparative fine structure of action: rules of form and sequence in the grooming patterns of six rodent species. Behaviour 113:21-56.

Berridge KC, Fentress JC (1986) Contextual control of trigeminal sensorimotor function. J Neurosci 6:325-330.

Black RG (1974) A laboratory model for trigeminal neuralgia. Adv Neurol 4:651-658.

Burchiel KJ (1980) Abnormal impulse generation in focally demyelinated trigeminal roots. J Neurosurg 53:674-683.

Cahusac PMB, Morris R, Salt TE, Hill RG (1990) Sensory responses of caudal trigeminal neurons to thermal and mechanical stimuli and their behavioural correlates in the rat. Neuroscience 36:543-551.

Cameron AA, Cliffer KD, Dougherty PM, Willis WD, Carlton SM (1991) Changes in lectin, GAP-43 and neuropeptide $Y$ in the rat superficial dorsal horn following experimental peripheral neuropathy. Neurosci Lett 131:249-252.

Campbell JN, Raja SN, Meyer RA (1988) Painful sequelae of nerve injury. In: Proceedings of the $\mathrm{V}$ th World Congress on Pain (Dubner R, Gebhart GF, Bond MR, eds), pp 135-143. Amsterdam: Elsevier.

Carlton SM, Dougherty PM, Pover CM, Coggeshall RE (1991) Neuroma formation and numbers of axons in a rat model of experimental peripheral neuropathy. Neurosci Lett 131:89-92.

Carvell GE, Simons DJ (1990) Biometric analyses of vibrissal tactile discrimination in the rat. J Neurosci 10:2638-2648.

Clavelou P, Pajot J, Dallel R, Raboisson P (1989) Application of the formalin test to the study of orofacial pain. Neurosci Lett 103:349353.

Coderre T, Grimes RW, Melzack R (1986) Deafferentation and chronic pain in animals: an evaluation of evidence suggesting autotomy is related to pain. Pain $26: 61-84$.

De Medinaceli L, Freed WJ, Wyatt RJ (1982) An index of the functional condition of rat sciatic nerve based on measurements made from walking tracks. Exp Neurol 77:634-643.

Devor M (1989) The pathophysiology of damaged peripheral nerves. In: Textbook of pain (Wall PD, Melzack R, eds), pp 63-81. London: Churchill-Livingston.

Devor M, Wall PD (1990) Cross-excitation in dorsal root ganglia of nerve injured and intact rats. J Neurophysiol 64:1733-1746.

Devor M, Schonfield D, Seltzer Z, Wall PD (1979) Two modes of cutaneous reinnervation following peripheral nerve injury. J Comp Neurol 185:211-220.

Draisci G, Kajander KC, Dubner R, Bennett GJ, Iadorola MJ (1991) Upregulation of opioid gene expression in spinal cord evoked by experimental nerve injuries and inflammation. Brain Res 560:186192.

Eisenberg E, Vos BP, Strassman AM (1993) The NMDA antagonist Memantine blocks pain behavior in a rat model of formalin-induced facial pain. Pain 54:301-307.

Fagen RM, Young DY (1978) Temporal patterns of behaviors: durations, latencies and sequences. In: Quantitative ethology (Colgan PW, ed), pp 79-114. New York: Wiley.

Fentress JC (1972) Development and patterning of movement sequences in inbred mice. In: The biology of behavior (Kiger $\mathbf{J}$, ed). Corvallis, OR: Oregon State University.

Fink BR, Aasheim G, Kish SJ, Croley TS (1975) Neurokinetics of lidocaine in the infraobital nerve of the rat in vivo. Anesthesiology 42:731-736.

Friedman $M$ (1937) The use of ranks to avoid the assumption of normality implicit in the analysis of variance. J Am Stat Assoc 32: 675-701.

Fromm GH, Sessle BJ (1991) Trigeminal neuralgia. Current concepts regarding pathogenesis and treatment. Boston: Butterworth-Heinemann.

Fromm GH, Terrence CF, Maroon JC. (1984) Trigeminal neuralgia, current concepts regarding etiology and pathogenesis. Arch Neurol 41:1204-1207.

Garrison CJ, Dougherty PM, Kajander KC, Carlton SM (1991) Staining of glial fibrillary acidic protein (GFAP) in lumbar spinal cord increases following a sciatic nerve constriction injury. Brain Res 565 : $1-7$.

Gautron M, Jazat F, Ratinahirana H, Hauw JJ, Guilbaud G (1990) Alterations in myelinated fibers in the sciatic nerve of rats after constriction: possible relationships between the presence of abnormal small myelinated fibres and pain-related behavior. Neurosci Lett 111: $28-33$.
Goodman LA, Kruskal WH (1979) Measures of association for crossclassifications. New York: Springer.

Greene EC (1955) Anatomy of the rat. New York: Hafner.

Gregg JM (1973) A surgical approach to the ophthalmic-maxillary nerve trunks in the rat. J Dent Res 52:392.

Gregg JM, Walter JM, Driscoll R (1979) Neurosensory studies of trigeminal dysesthesia following trigeminal nerve injury. In: Advanced pain research therapy, Vol 3 (Bonica JJ, Liebeskind JC, AlbeFessard DG, eds), pp 311-315. New York: Raven.

Gregoire SE, Smith DE (1975) Mouse-killing in the rat: effects of sensory deficits on attack behaviour and stereotyped biting. Anim Behav 23:186-191.

Grey JA, Lalljee B (1974) Sex differences in emotional behaviour in the rat: correlation between open field defecation and active avoidance. Anim Behav 22:856-861.

Griswold JG, Borchelt PL, Branchek RS, Bensko JA (1977) Condition of the pelage regulates sand bathing and grooming behaviour in the kangaroo rat (Dipodomys merriami). Anim Behav 25:602-608.

Guilbaud G, Benoist JM, Jazat F, Gautron M (1990) Neuronal responsiveness in the ventrobasal complex of rats with an experimental peripheral mononeuropathy. J Neurophysiol 64:1537-1557.

Guilbaud G, Benoist JM, Levante A, Gautron M, Willer JC (1992) Primary somatosensory cortex in rats with pain-related behaviours due to a peripheral mononeuropathy after moderate ligation of one sciatic nerve: neuronal responsitivity to somatic stimulation. Exp Brain Res 92:227-245.

Hinkle DE, Oliver JD (1983) How large should the sample be? A question with no simple answer? Or. . . Educ Psychol Measurement 43:1051-1060.

Hirata H, Pataky A, Kajander K, LaMotte RH, Collins JG (1990) A model of peripheral mononeuropathy in the rat, letter to the editor. Pain 42:253-254.

Hofer MA, Fisher A, Shair H (1981) Effects of infraorbital nerve section on survival, growth, and suckling behaviors of developing rats. J Comp Physiol Psychol 95:123-133.

Huston JP, Steiner H, Weiler H-T, Morgan S, Schwarting RKW (1990) The basal ganglia-orofacial system: studies on neurobehavioral plasticity and sensory-motor tuning. Neurosci Biobehav Rev 14:433-446.

International Association for the Study of Pain (1983) Ethical guidelines for investigations of experimental pain in conscious animals. Pain 16:109-110.

Jacquin MF, Zeigler HP (1983) Trigeminal orosensation and ingestive behavior in the rat. Behav Neurosci 97:62-97.

Jacquin MF, Zeigler HP (1984) Trigeminal denervation and operant behavior in the rat. Behav Neurosci 98:1004-1022.

Jacquin MF, Hess A, Yang G, Adamo P, Math MP, Brown A, Rhoades RW (1984) Organization of the infraorbital nerve in rat: a quantitative electron microscopic study. Brain Res 290:131-135.

Jacquin MF, Chiaia NL, Rhoades RW (1990) Trigeminal projections to contralateral dorsal horn: central extent, peripheral origins, and plasticity. Somatosens Motor Res 7:153-183.

Kajander KC, Bennett GJ (1992) Onset of a painful peripheral neuropathy in rat: a partial and differential deafferentation and spontaneous discharge in $\mathrm{A} \beta$ and A-delta primary afferent neurons. J Neurophysiol 68:734-744.

Kajander KC, Sahara Y, Iadarola MJ, Bennett GJ (1990a) Dynorphin increases in the dorsal spinal cord in rats with a painful peripheral neuropathy. Peptides 11:719-728.

Kajander KC, Wakisaka S, Driasci G, Iadarola MJ (1990b) Labeling of fos protein increases in an experimental model of peripheral neuropathy in the rat. Soc Neurosci Abstr 16:1281.

Kajander KC, Wakisaka S, Bennett GJ (1992) Spontaneous discharge originates in the dorsal root ganglion at the onset of a painful peripheral neuropathy in the rat. Neurosci Lett 138:225-228.

Kenyon P, Cronin P, Keeble S (1983) Role of the infraorbital nerve in retrieving behavior in lactating rats. Behav Neurosci 97:255-269.

Kerns JM, Braverman B, Mathew A, Lucchinetti C, Ivankovich AD (1991) A comparison of cryoprobe and crush lesions in the rat sciatic nerve. Pain 47:31-39.

Kerr FWL (1970) Peripheral versus central factors in trigeminal neuralgia. In: Trigeminal neuralgia, pathogenisis und pathophysiology (Hassler R, Walker AE, eds), pp 180-190. Stuttgart: Thicmc.

Kim SH, Chung JM (1992) An experimental model for peripheral neuropathy produced by segmental nerve ligation in the rat. Pain 50 : $355-363$.

King RB (1970) Electrophysiology of trigeminal neurons under normal and epileptogenic conditions. In: Trigeminal neuralgia, pathogenesis 
und pathophysiology (Hassler R, Walker AE, eds), pp 78-85. Stuttgart: Thieme.

King RB, Barnett JCB (1957) Studies of trigeminal nerve potentials: overreaction to tactile facial stimulation in acute laboratory preparations. J Neurosurg 14:617-627.

King RB, Meagher JN, Barnett JC (1956) Studies of trigeminal nerve potentials: overreaction to tactile facial stimulation in acute laboratory preparations. J Neurosurg 13:167-183.

Klein BG, Misra BR, White CF (1991) Orofacial pain sensitivity in adult rats following neonatal infraorbital nerve section. Behav Brain Res 41:197-201.

Kryzhanovsky GN, Igonkina SI, Grafova VN, Danilova EI (1974) Experimental trigeminal neuralgia (on the conception of the generator mechanism of the pain syndrome). Biull Eksp Biol Med 78:16-20.

Kugelberg E, Lindblom $U$ (1959) The mechanism of the pain in trigeminal neuralgia. J Neurol Neurosurg Psychiatry 22:36-43.

Kupers RC, Nuytten D, De Castro-Costa M, Gybels JM (1992) A time course analysis of the changes in spontaneous and evoked behaviour in a rat model of neuropathic pain. Pain 50:101-111.

Laird JMA, Bennett GJ (1992) Dorsal root potentials and afferent input to the spinal cord in rats with an experimental peripheral neuropathy. Brain Res 584:181-190.

Laird JMA, Bennett GJ (1993) An electrophysiological study of dorsal horn neurons in the spinal cord of rats with an experimental peripheral neuropathy. J Neurophysiol 69:2072-2085.

Lindblom U (1990) Classification and assessment of altered sensation and pain. In: Altered sensation and pain (Dimitrijevic MR, Wall PD, Lindblom U, eds), pp 7-16. Basel: Karger.

Lindblom U, Hansson P (1991) Sensory dysfunction and pain after clinical nerve-injury studied by means of graded mechanical and thermal stimulation. In: Lesions of primary afferent fibers as a tool for the study of clinical pain (Besson JM, Guilbaud G, eds), pp 1-18. Amsterdam: Elsevier.

Lindblom U, Verrillo RT (1979) Sensory functions in chronic neuralgia. J Neurol Neurosurg Psychiatry 42:422-435.

Lombard MC, Nashold BS, Albe-Fessard D (1979) Deafferentation hypersensitivity in the rat after dorsal rhizotomy: possible animal model for chronic pain. Pain 6:163-174.

Merskey H (1986) Pain terms. Pain [Suppl] 3:215-221.

Milani H, Steiner H, Huston JP (1989) Analysis of recovery of behavioral asymmetrics induced by unilateral removal of vibrissae in the rat. Behav Neurosci 103:1067-1074.

Miller MG (1981) Trigeminal deafferentation and ingestive behavior in the rat. J Comp Physiol Psychol 95:252-269.

Mitchell SW (1872) Injuries of nerves and their consequences. Philadelphia: Lippincott.

Morris R, Cahusac PMB, Morris RG, Hill RG (1982) A behavioral model for the study of facial nociception and the effects of descending modulatory systems in the rat. J Neurosci Methods 6:245-252.

Morton DB, Griffiths PHM (1985) Guidelines on the recognition of pain, distress and discomfort in experimental animals and an hypothesis for assessment. Vet Rec 116:431-436.

Munger BL, Bennett GJ, Kajander KC (1992) An experimental painful peripheral neuropathy due to nerve constriction. I. Axonal pathology in the sciatic ncrve. Exp Neurol 118:204-214.

National Research Council (1992) Recognition and alleviation of pain and distress in laboratory animals. A report of the Committee on Pain and Distress in Laboratory Animals, Institute of Laboratory Animal Resources, Commission on Life Sciences, National Research Council. Washington, DC: National Academy Press.

Noordenbos W (1959) Pain. Amsterdam: Elsevier.

O'Kelly LI, Steckle LC (1939) A note on long enduring emotional responses in the rat. J Psychol 8:125-131.

Palecek J, Paleckova V, Dougherty PM, Carlton SM, Willis WD (1992) Responses of spinothalamic tract cells to mechanical and thermal stimulation of skin in rats with experimental peripheral neuropathy. J Neurophysiol 67:1562-1573.

Ramabadran K, Bansinath M (1986) A critical analysis of the experimental evaluation of nociceptive reactions in animals. Pharmacol Res Rev 3:263-270.

Ro L-S, Jacobs JM (1993) The role of the saphenous nerve in experimental sciatic nerve mononeuropathy produced by loose ligatures: a behavioural study. Pain 52:359-369.

Rosenfeld JP, Broton JG, Clavier RM (1978) A reliable, facial nociception device for unrestrained, awake animals: effects of morphine and trigeminal complex lesions. Physiol Behav 21:287-290.
Sakai Y, Nishijima Y, Mikuni N, Iwata N (1979) An experimental model of hyperirritability in the trigeminal skin field of the rat. Pain 7:147-157.

Schouenborg J, Kalliomäki J (1990) Functional organization of the nociceptive withdrawal reflexes: 1 . Activation of hind limb muscles in the rat. Exp Brain Res 83:67-87.

Seltzer Z, Dubner R, Shir Y (1990) A novel behavioral model of neuropathic pain disorders produced in rats by partial sciatic nerve injury. Pain 43:205-218.

Sherrington CS (1910) Flexion-reflex of the limb, crossed extensionreflex and reflex stepping and standing. J Physiol (Lond) 40:28-121.

Stern JM, Kolunie JM (1991) Trigeminal lesions and maternal behavior in Norway rats: I. Effects of cutaneous rostral snout denervation on maintenance of nurturance and maternal aggression. Behav Neurosci 105:984-997.

Stevens CW, Kajander KC, Bennett GJ, Seybold VS (1991) Bilateral and differential changes in spinal mu, delta, and kappa opioid binding in rats with a painful, unilateral neuropathy. Pain 46:205-213.

Sugimoto T, Bennett GJ, Kajander KC (1990) Transsynaptic degeneration in the superficial dorsal horn after sciatic nerve injury: effects of a chronic constriction injury, transection, and strychnine. Pain 42: 205-213.

Sweet WH (1984) Deafferentation pain after posterior rhizotomy, trauma to a limb and herpes zoster. Neurosurgery 15:1984.

Tarapacki JA, Kristal MB (1990) An adaptable microcomputer program for recording behavior durations. Physiol Behav 47:381-384.

Tomie J, Whishaw IQ (1990) New paradigms for tactile discrimination studies with the rat: methods for simple, conditional and configurational discriminations. Physiol Behav 48:225-231.

Vincent SB (1913) The tactile hair of the white rat. J Comp Neurol 23:1-36.

Vos BP, Maciewicz RJ (1990) Alterations of peripheral nerve morphology and changes in fluoride resistant acid phosphatase and substance P-immunoreactivity in the trigeminal nucleus caudalis in an experimental model of trigeminal neuropathic pain. Am Pain Soc Abstr 9:56.

Vos BP, Maciewicz RJ (1991) Behavioral changes following ligation of the infraorbital nerve in rat: an animal model of trigeminal neuropathic pain. In: Lesions of primary afferent fibers as a tool for the study of clinical pain (Besson JM, Guilbaud G, eds), pp 147-158. Amsterdam: Elsevier.

Vos BP, Strassman AM, Maciewicz RJ (1991) Effects of loose ligation of the infraorbital nerve on c-fos expression in the medullary dorsal horn of the rat. Soc Neurosci Abstr 17:437.

Wakisaka S, Kajander KC, Bennett GJ (1992) Immunohistochemical analysis of the changes in neuropeptide $\mathrm{Y}$ following peripheral nerve injury and local inflammation. In: Processing and inhibition of nociceptive information (Inoki R, Shigenaga $Y$, Tohyama M, eds), pp 157-160. Amsterdam: Elsevier.

Wall PD, Devor M, Inbal R, Scadding JW, Schonfeld D, Seltzer Z, Tomkiewicz MM (1979) Autotomy following peripheral nerve lesions: experimental anaesthesia dolorosa. Pain 7:103-113.

Welle SL, Coover GD (1978) Deficits in mouse-killing following trigeminal lesions in the rat. Physiol Psychol 6:332-339.

White JC, Sweet WH (1969) Pain and the neurosurgeon. Springfield, IL: Thomas.

Woolf CJ, Shortland P, Coggeshall RE (1992) Peripheral nerve injury triggers central sprouting of myelinated afferents. Nature 355:75-78.

Xie Y, Xiao W-H (1990) Electrophysiological evidence for hyperalgesia in the peripheral neuropathy. Sci China Ser B Chem Life Sci Earth Sci 33:663-672.

Yaksh TL (1989) Behavioral and autonomic correlates of the tactile evoked allodynia produced by spinal glycine inhibition: effects of modulatory receptor systems and excitatory amino acid antagonists. Pain 37:111-123.

Yaksh TL, Yamamoto T, Myers RR (1992) Pharmacology of nerve compression-evoked hyperesthesia. In: Hyperalgesia and allodynia (Willis WD, ed), pp 245-258. New York: Raven.

Yamamoto T, Yaksh TL (1992) Spinal pharmacology of thermal hyperesthesia induced by constriction injury of sciatic nerve. Excitatory amino acid antagonists. Pain 49:121-128.

Zeigler HP, Semba K, Jacquin MF (1984) Trigeminal reflexes and ingestive behavior in the rat. Behav Neurosci 98:1023-1038. 